\title{
Generalized Collapse Solutions with Nonzero Initial Velocities for Star Formation in Molecular Cloud Cores
}

\author{
Marco Fatuzzo, ${ }^{1}$ Fred C. Adams, ${ }^{2,3}$ and Philip C. Myers ${ }^{4}$ \\ ${ }^{1}$ Physics Department, Xavier University, Cincinnati, OH 45207 \\ ${ }^{2}$ Michigan Center for Theoretical Physics, University of Michigan \\ Physics Department, Ann Arbor, MI 48109 \\ ${ }^{3}$ Astronomy Department, University of Michigan, Ann Arbor, MI 48109 \\ ${ }^{4}$ Harvard Smithsonian Center for Astrophysics, 60 Garden Street, Cambridge, MA 02138 \\ fatuzzo@cerebro.cs.xu.edu, fca@umich.edu, pmyers@cfa.harvard.edu
}

\begin{abstract}
Motivated by recent observations that show starless molecular cloud cores exhibit subsonic inward velocities, we revisit the collapse problem for polytropic gaseous spheres. In particular, we provide a generalized treatment of protostellar collapse in the spherical limit and find semi-analytic (self-similar) solutions, corresponding numerical solutions, and purely analytic calculations of the mass infall rates (the three approaches are in good agreement). This study focuses on collapse solutions that exhibit nonzero inward velocities at large radii, as observed in molecular cloud cores, and extends previous work in four ways: (1) The initial conditions allow nonzero initial inward velocity. (2) The starting states can exceed the density of hydrostatic equilibrium so that the collapse itself can provide the observed inward motions. (3) We consider different equations of state, especially those that are softer than isothermal. (4) We consider dynamic equations of state that are different from the effective equation of state that produces the initial density distribution. This work determines the infall rates over a wide range of parameter space, as characterized by four variables: the initial inward velocity $v_{\infty}$, the overdensity $\Lambda$ of the initial state, the index $\Gamma$ of the static equation of state, and the index $\gamma$ of the dynamic equation of state. For the range of parameter space applicable to observed cores, the resulting infall rate is about a factor of two larger than found in previous theoretical studies (those with hydrostatic initial conditions and $v_{\infty}=0$ ).
\end{abstract}

Subject headings: hydrodynamics - stars: formation 


\section{INTRODUCTION}

Stars form through gravitational collapse and hence the dynamics of the collapse flow is fundamental to understanding the star formation process. Historically, theoretical studies of gravitational collapse have considered initial conditions that correspond to hydrostatic equilibrium (or near equilibrium). In recent years, however, observations have shown that some molecular cloud cores display nonzero, inward velocities before they contain infrared sources. One standard interpretation of this finding is that the cores must begin their collapse with a head start, i.e., with a pre-existing inward flow. The goal of this paper is to reexamine the gravitational collapse problem for star formation with a focus on initial conditions that include initial inward flows, i.e., the conditions observed in star forming regions. Along the way, we generalize previous studies of protostellar collapse to include a wider set of equations of state.

To isolate the effects of the inward flow, we return to the basic spherical collapse problem for polytropic spheres, which are used as idealized theoretical models (Shu 1977) of observed molecular cloud cores (e.g., Myers \& Benson 1983, Benson \& Myers 1987). This same treatment can be applied to the collapse of subcondensations (denoted as kernels - see Myers 1998) within larger cores that form stellar groups. In the simplest scenario, molecular cloud cores are thought to evolve into centrally concentrated configurations through the process of ambipolar diffusion (Mouschovias 1976; Shu 1983; Nakano 1984). Since recent observations suggest that core formation takes place more rapidly than predicted by standard models of ambipolar diffusion (e.g., Jijina, Myers, \& Adams 1999), several modifications to this picture have been suggested. These generalizations include ambipolar diffusion starting near the supercritical state (Ciolek \& Basu 2001), turbulent fluctuations speeding up ambipolar diffusion rates (Fatuzzo \& Adams 2002; Zweibel 2002), core formation through turbulent cooling flows (Myers \& Lazarian 1998), and core (and star) formation through dynamic, turbulent fragmentation (Li, Klessen, \& MacLow 2003; see also Li 1999). In the extreme limit of some efficient fragmentation scenarios, pre-collapse states (cores) never actually form at all. Our present approach - studying the collapse of cores that form with a pre-existing inward flow - is intermediate between the previous picture of star formation starting from hydrostatic equilibrium (Shu, Adams, \& Lizano 1987) and the alternate scenario of a completely dynamic star formation process (MacLow \& Klessen 2003). In any case, molecular cloud cores are observed to form relatively rapidly and to (often) exhibit subsonic inward motions in their starless state (Lee et al. 1999, 2001). These configurations thus specify the initial conditions for star formation and their subsequent collapse determines the manner in which stars form.

The collapse of cloud cores can be studied using self-similar methods. Indeed, self-similar collapse solutions (Shu 1977) are an important cornerstone of the current theory of star formation (e.g., Shu et al. 1987). The original self-similar collapse calculations (Larson 1969ab; Penston 1969ab; Shu 1977) considered an isothermal equation of state. Since then, many generalizations of the collapse have been made. The leading order effects of rotation have been studied, both for the inner pressure free region (Ulrich 1976; Cassen \& Moosman 1981) and for the entire core (Terebey, Shu, \& Cassen 1984). Different equations of state for the collapsing gas have been studied 
(Cheng 1977, 1978; McLaughlin \& Pudritz 1997). The leading order effects of magnetic fields have also been included (Galli \& Shu 1993ab; Li \& Shu 1996, 1997). More recently, the collapse of magnetized singular isothermal toroids has been studied (Allen, Shu, \& Li 2003; Allen, Li, \& Shu 2003); the latter study includes a calculation of collapse with an imposed initial velocity (see their Figure 7), in the same spirit as this investigation. The effects of radiation pressure on the collapse flow have been studied in both the spherical limit (e.g., Wolfire \& Cassinelli 1986, 1987) and the inner pressure free regime for rotating collapse (Jijina \& Adams 1996). Additional mathematical generalizations of the solutions have also been considered (Hunter 1977; Whitworth \& Summers 1985).

The self-similar collapse solutions explored in this paper are applicable for cores that exhibit a wide range of densities. Actual molecular cloud cores have a finite central density $n_{C}$ that extends over a given radial extent $r_{C} \sim a_{S}\left(2 \pi G \mu n_{C}\right)^{-1 / 2}$. The core must also have an outer radial scale $r_{\text {out }}$ where the declining density profile of the core matches onto the background of the molecular cloud. If $r_{M}$ denotes the radius that encloses a stellar mass, then self-similar solutions apply over the ordering of radial scales $r_{C} \ll r_{M} \ll r_{\text {out }}$. Previous numerical work (Foster \& Chevalier 1993) indicates that the collapse of an isothermal core approaches the expected self-similar form when the core has $r_{\text {out }} / r_{C}>20$. For typical values $M=0.5 M_{\odot}$ and $a_{S}=0.2 \mathrm{~km} / \mathrm{s}$, this criterion implies a constraint on the central number density $n_{C}>3 \times 10^{7} \mathrm{~cm}^{-3}$. In the context of this paper, however, cores that have initial inward velocities can more readily approach the self-similar collapse forms and these constraints $\left(r_{\text {out }} / r_{C}>20 ; n_{C}>3 \times 10^{7} \mathrm{~cm}^{-3}\right)$ are less severe.

Only a fraction of the observed starless cores have a sufficiently extended range of densities (or, equivalently, large enough ratios $r_{\text {out }} / r_{C}$ ) so that the collapse flows can be modeled to high accuracy with the self-similar forms derived here. Many observed cores have flat central regions (Tafalla et al. 2004), and tend to approach the form $\rho \sim r^{-2}$ beyond a few thousand AU (Ward-Thompson, Motte, \& André 1999). In a recent survey (Bacmann et al. 2000), 3 of the 24 observed cores also show evidence for a well defined edge (corresponding to the scale $r_{\text {out }}$ ) so that these cores have small ratios $r_{\text {out }} / r_{C}$. However, mapping results in Taurus are consistent with a self-similar collapse picture and the envelopes in Bok globules are also qualitatively consistent (Motte \& André 2001). For cores with constant density centers, the infall rate will display more time variability than in the self-similar solutions: The infall rate will be very small at first, but quickly grows to become larger than that of the self-similar solution; once the constant density portion of the core has collapsed, the infall rate decreases toward the self-similar value (e.g., Foster \& Chevalier 1993; Myers 2004). Furthermore, even if actual cores only approach the self-similar solutions asymptotically in time, this idealized formulation of the collapse problem allows us to isolate the effects of various physical inputs (especially nonzero initial velocities).

The overall goal of this paper is thus to obtain a greater understanding of the collapse of molecular cloud cores. This work includes four generalizations of previous studies of protostellar collapse, but the most important astronomical issue is to include the effects of pre-existing inward flows, which have now been observed (as outlined above). We can include an initial inward velocity 
as part of the initial conditions (i.e., we let $v \neq 0$ at large radii). In a similar vein, we can consider initial states that are overdense and thus exceed the density required for hydrostatic equilibrium. These two complications are related in that both can explain the observed inward flows and both lead to larger mass infall rates, but they are conceptually distinct. In the first case, the inward flows are part of the initial conditions and must be produced by whatever physical processes form the core. In the case of overdense initial states, however, the inward flows are part of the collapse. Specifically, in the latter case, the core begins with zero initial velocity throughout its structure, but the overdense core soon develops small, but nonzero, inward speeds at large radii (as observed).

In addition, we consider equations of state that are softer than isothermal. This generalization is motivated by observations of non-thermal linewidths $\Delta v$ in molecular cloud cores, where one finds correlations with density of the form $(\Delta v)^{2} \sim \rho^{-\beta}$, with $\beta>0$ (e.g., Larson 1985, Jijina et al. 1999). If one interprets the linewidth $\Delta v$ as the effective transport speed in the medium, then the corresponding effective equation of state has the form $P \sim \rho^{\Gamma}$, where $\Gamma=1-\beta$ and hence $0<\Gamma \leq 1$. In the limit $\Gamma \rightarrow 0, P \rightarrow \log \rho$; this limiting case of a sequence of possible equations of state is often called a "logatrope" (beginning with Lizano \& Shu 1989). Here we consider a range of effective equations of state, from the isothermal limit $\Gamma=1$ down to the logatropic limit $\Gamma \rightarrow 0$ (collapse solutions in the logatropic limit have been studied previously by McLaughlin \& Pudritz 1997). Notice that the general equations we work with below reduce to the logatropic case in the limit $\Gamma \rightarrow 0$ (but care must be exercised in taking the limit).

As another generalization, we consider the case in which the dynamic equation of state for the collapsing gas is different than the effective (static) equation of state that produces the initial equilibrium configuration. Here, the static equation of state refers to the pressure law that enforces the initial (pre-collapse) configuration for the core. Note that we consider only barotropic equations of state throughout this paper. The dynamic equation of state (as set by $\gamma$ ) refers to the pressure law that describes how the thermodynamic variables of the gas change as the material is compressed during collapse; this process is governed by the entropy evolution equation (see $\S 2.1$ ). Equations of state that are softer than isothermal are motivated by the observed linewidth dependence on density, and the inferred density profiles, in molecular cloud regions. These considerations apply primarily to the static equation of state as defined here (for applications of such soft, static equations of state, see, e.g., Curry \& McKee 2000, Curry \& Stahler 2001, Spaans \& Silk 2000). The dynamic equation of state, as set by the index $\gamma$ that appears in equation (5), need not be the same as the static equation of state. In other words, the physics that determines the density profiles of the pre-collapse states can be different from the physics that governs the thermodynamics of the collapse flow. We thus allow $\gamma \neq \Gamma$. This possibility is also motivated by theoretical MHD simulations (VazquezSemadeni, Canto, \& Lizano 1998) which show that the turbulent velocity dispersion increases with mean density as the gas collapses, in contradiction to the expected behavior for a soft equation of state; as a result, logatropic and similarly soft equations of state may provide an inadequate description of the dynamical processes occurring within a cloud.

The mass infall rate $\dot{M}$ is one of the most important physical quantities in the star formation 
problem and this paper focuses on calculating $\dot{M}$ for a range of conditions. Collapse flows are often self-similar and have no characteristic mass scale. Instead, the collapse flow feeds material onto the central star/disk system at a well-defined mass infall rate $\dot{M}$. In these flows, the infalling material always approaches free-fall conditions on the inside (in the limit $r \rightarrow 0$ ) and the reduced mass $m_{0}$ determines the size of the infall rate (as shown below). The infall rate $\dot{M}$ determines, in part, the total system luminosity and the total column density of the infalling envelope. These quantities, in turn, largely account for the spectral appearance of protostellar objects (e.g., Adams, Lada, \& Shu 1987; Adams 1990). For protostellar objects, most of the luminosity is derived from material falling through the gravitational potential well of the star. Although the circumstellar disk stores some of the energy in rotational motion, the system luminosity is (usually) a substantial fraction of the total available luminosity

$$
L_{0} \equiv \frac{G M \dot{M}}{R_{*}}
$$

where $M$ is the total mass of the system, $\dot{M}$ is the mass infall rate, and $R_{*}$ is the stellar radius. The stellar radius, which helps determine the depth of the potential well, is itself a function of the mass infall rate (Stahler, Shu, \& Taam 1980).

This paper is organized as follows. In $\S 2$, we present a general formulation of the collapse problem, including both the similarity transformation for self-similar solutions and a complementary numerical treatment. We present general analytic results in $\S 3$, including a discussion of how the "outer" spherically symmetric solutions of this paper match onto collapse solutions in the inner regime where the effects of rotation are important. In $\S 4$, we consider collapse solutions for which the static equation of state is isothermal (so that the initial state $\rho \sim r^{-2}$ ), but generalize previous

work to include nonzero initial flow speeds $\left(v_{\infty}>0\right)$, overdense initial configurations $(\Lambda>1)$, and dynamic equations of state that are not isothermal $(\Gamma=1, \gamma \neq 1)$. In $\S 5$ we consider collapse solutions from non-isothermal initial conditions, i.e., starting states for which the static $\Gamma \neq 1$. In $\S 6$, we conclude with a summary and discussion of our results.

\section{FORMULATION OF THE COLLAPSE PROBLEM}

In this section we formulate the collapse problem for the general case of a collapsing spherical cloud of gas. The collapse solution is obtained by self-similar methods ( $\S 2.2)$ and by a numerical approach (§2.3). In both cases, we allow the initial states to be out of exact hydrostatic equilibrium by having initial inward velocities and/or by being overdense. We also allow the dynamic equation of state to be different from the static equation of state that determines the initial density distribution. 


\subsection{Basic Governing Equations}

The basic equations governing the fluid are given below. We want to consider the spherical collapse problem, so that the equations of motion reduce to a simpler form. Conservation of mass can be expressed in terms of mass shells

$$
\frac{\partial M}{\partial t}+u \frac{\partial M}{\partial r}=0 \quad \text { and } \quad \frac{\partial M}{\partial r}=4 \pi r^{2} \rho
$$

where $M(r, t)$ is the total mass within a given radius $r$ at a time $t$. The above equations are equivalent to the usual equation of continuity,

$$
\frac{\partial \rho}{\partial t}+\frac{1}{r^{2}} \frac{\partial}{\partial r}\left(r^{2} \rho u\right)=0
$$

where $\rho$ is the density and $u$ is the radial component of the velocity. The force equation can be written in the form

$$
\frac{\partial u}{\partial t}+u \frac{\partial u}{\partial r}=-\frac{1}{\rho} \frac{\partial P}{\partial r}-\frac{G M}{r^{2}},
$$

where $P$ is the pressure. Finally, the conservation of entropy equation can be written in the form

$$
\left(\frac{\partial}{\partial t}+u \frac{\partial}{\partial r}\right)\left[\log \left(P / \rho^{\gamma}\right)\right]=0
$$

where $\gamma$ is the index of the dynamic equation of state. Adopting equation (5) is not the same as assuming an equation of state of the form $P=K \rho^{\gamma}$. Equation (5) describes how a given parcel of gas changes its thermodynamic variables along a streamline, whereas the equation of state $\left(P=K \rho^{\gamma}\right)$ implies a global constraint on those variables.

\subsection{The Similarity Transformation}

In this section, we find a similarity transformation for the collapse problem formulated above. Mathematically, this problem is represented by coupled partial differential equations in the variables

time $t$ and radial position $r$. Using the similarity transformation, we can reduce this problem to a set of ordinary differential equations in a new similarity variable $x$ which we define below. In particular, we look for a similarity transformation of the general form

$$
\begin{gathered}
x=A t^{a} r, \quad \rho=B t^{b} \alpha(x), \quad M=C t^{c} m(x), \\
u=D t^{d} v(x), \quad \text { and } \quad P=E t^{e} p(x) .
\end{gathered}
$$

Here, both the coefficients $(A, B, C, D, E)$ and the indices $(a, b, c, d, e)$ are constants. The reduced fluid fields $(\alpha, m, v, p)$ are dimensionless functions of the (single) dimensionless similarity variable $x$. The time benchmark $t=0$ corresponds to the instant of the onset of collapse when the mass of the central "object" is zero, i.e., $M(0, t)=0$. 
The general similarity transformation calculation leads to four equations to specify the five indices $a, b, c, d, e$. We leave the constant $a$ arbitrary for the moment and write the rest of the variables in terms of its value, i.e.,

$$
\begin{gathered}
a=a, \quad b=-2, \quad c=-(3 a+2), \\
d=-(a+1), \quad \text { and } \quad e=-2(a+2) .
\end{gathered}
$$

Similarly, for the coefficients we obtain

$$
\begin{gathered}
A=A, \quad B=(4 \pi G)^{-1}, \quad C=\left(A^{3} G\right)^{-1}, \\
D=A^{-1}, \quad \text { and } \quad E=\left(4 \pi G A^{2}\right)^{-1} .
\end{gathered}
$$

Keep in mind that $G$ is the gravitational constant. We thus obtain reduced equations of motion in the form

$$
\begin{gathered}
(a x+v) \frac{d m}{d x}=(3 a+2) m, \\
\frac{d m}{d x}=x^{2} \alpha \\
(a x+v) \frac{d v}{d x}+\frac{1}{\alpha} \frac{d p}{d x}=-\frac{m}{x^{2}}+(a+1) v, \\
(a x+v) \frac{d}{d x} \log \left[p / \alpha^{\gamma}\right]=2(2+a-\gamma), \\
(a x+v) \frac{1}{\alpha} \frac{d \alpha}{d x}+\frac{d v}{d x}=2(1-v / x) .
\end{gathered}
$$

This similarity transformation is not unique - one can always rescale the coefficients $\{A, B, C, D, E\}$ by a set of dimensionless numbers and obtain new equations of motion with different numerical coefficients.

Notice that we can immediately combine the first two equations of motion to obtain an expression for the reduced mass $m(x)$, i.e.,

$$
m=\frac{(a x+v)}{(3 a+2)} x^{2} \alpha .
$$

Next we define a constant $q$ according to

$$
q \equiv \frac{2}{3 a+2}(2+a-\gamma)
$$

If we then take $q$ times equation (9) and subtract it from equation (12), we can integrate the resulting equation to obtain an expression for the reduced pressure $p(x)$, i.e.,

$$
p=\mathcal{C}_{0} \alpha^{q+\gamma}\left(x^{3}+v x^{2} / a\right)^{q}=\mathcal{C}_{1} \alpha^{\gamma} m^{q},
$$

where $\mathcal{C}_{0}$ and $\mathcal{C}_{1}$ are constants which depend on the initial density distribution of the core. Given these solutions for the reduced pressure $p(x)$ and reduced mass $m(x)$, equations (11) and (13) are 
the relevant equations of motion to determine the remaining unknown functions $\alpha(x)$ and $v(x)$. Notice that in the limit $x \rightarrow \infty$, the solution (16) for the pressure approaches the form $p \rightarrow C_{2} \alpha^{\Gamma}$ (obtained by integrating the equations of motion). In the opposite limit $x \rightarrow 0, p \rightarrow C_{3} \alpha^{\gamma}$. In other words, this solution for the pressure illustrates how the equation of state smoothly transforms from static index $\Gamma$ (at early times and/or large radii) to the dynamic index $\gamma$ (at late times and/or small radii).

In this paper, we consider only positive values of time, since we take the zero point of the time variable to be the onset of collapse. It is mathematically possible to consider solutions for negative times, $t<0$, which would correspond to negative values of $x$. Solutions that span the entire available range $-\infty<x<\infty$ are known as "complete" solutions (first obtained by Hunter 1977; see also Whitworth \& Summers 1985). Note that the self-similar solutions of this paper for the protostellar collapse phase $(t>0)$ cannot (in general) be extended to the pre-stellar phase $(t<0)$, as they would likely encounter a critical point and become singular. In other words, dynamical evolution (using only collapse physics) in the $t<0$ regime would not (in general) lead to the initial conditions used here. However, it is rather unlikely that molecular clouds will evolve toward their centrally condensed initial configurations in a self-similar manner subject to (only) the physics included in these equations of motion. Before the onset of collapse (for $t<0$ ), molecular clouds may evolve through the processes of ambipolar diffusion (at least for small mass scales), shocks, turbulent dissipation, cooling flows, condensation instabilities, and cloud-cloud collisions. In addition, the cloud will most likely initiate collapse before a completely self-similar equilibrium state has been attained; the collapse will only become self-similar asymptotically in time (i.e., the self-similar collapse solutions of this paper are intermediate asymptotic solutions to the realistic problem of the collapse of a finite cloud with finite central density). In any case, we limit this discussion to solutions with $0<x<\infty$, sometimes called "semi-complete" solutions.

\subsection{Numerical Treatment}

As a complement to the self-similar formulation, we also perform numerical simulations of the collapse for the case where $\Gamma=\gamma=1$. For the isothermal equations of state considered here, $P=a_{s}^{2} \rho$, the force equation can be written as

$$
\frac{\partial}{\partial t}(\rho u)+\frac{1}{r^{2}} \frac{\partial}{\partial r}\left(r^{2} \rho u^{2}\right)=-a_{s}^{2} \frac{\partial \rho}{\partial r}-\rho \frac{G M}{r^{2}} .
$$

This form of the force equation is solved numerically along with equation (3) using the secondorder-accurate scheme outlined in Boss \& Myhill (1992). This scheme invokes a variation on the standard predictor-corrector technique, and allows for nonuniform grid spacing. Our grid is defined by 160 logrithmically spaced radii $\left(r_{i}\right)$ spanning four orders of magnitude from the inner to the outer boundaries. The cell interfaces $\left(r_{i \pm 1 / 2}\right)$ are then set at the midway points of these radii. The mass terms at each radius $\left(M_{i}\right)$ are advanced in time (denoted by the superscript $n$ ) using the 
scheme

$$
M_{i}^{n+1}=M_{i}^{n}-F_{i}^{n} 4 \pi r_{i}^{2} \Delta t,
$$

where the flux terms are defined as

$$
F_{i}^{n}=\rho_{i}^{n} v_{i}^{n}+\frac{\Delta t}{2}\left[\rho_{i}^{n}\left(\frac{\partial u}{\partial t}\right)_{i}^{n}+u_{i}^{n}\left(\frac{\partial \rho}{\partial t}\right)_{i}^{n}\right],
$$

and the right hand terms in the above equations are defined in Boss \& Myhill (1992).

\section{GENERAL ANALYTIC RESULTS}

Given the formulation of the collapse problem, we now find several analytic results. These results are general in that they apply to the entire class of solutions considered in this paper.

\subsection{Relationship Between the Similarity Transformation and the Static Equation of State}

First, we show that the initial density profile of the core determines the type of similarity transformation which describes the subsequent collapse of the core. To start, we assume that the initial equilibrium configuration of the system can be described by a "static" equation of state of the form

$$
P=\kappa \rho^{\Gamma} .
$$

Since the similarity transformation determines the manner in which both the pressure $P$ and the density $\rho$ must scale with time, this new equation of state imposes an additional constraint on the

system. In particular, the similarity transformation requires that the scaling exponent $a$ (which has been left arbitrary thus far), must have the value

$$
a=\Gamma-2,
$$

where the similarity variable is defined by $x=A t^{a} r$. We also obtain a required value for the corresponding numerical coefficient $A$, i.e.,

$$
A=\kappa^{-1 / 2}(4 \pi G)^{(\Gamma-1) / 2} .
$$

With this choice for $A$, the reduced static equation of state has the form $p=\alpha^{\Gamma}$. As mentioned before, however, this coefficient $A$ can be rescaled by a dimensionless constant without changing the physics (as long as that constant is propagated throughout the set of equations of motion).

Notice that for the special case in which the static and dynamic equations of state are the same (i.e., $\Gamma=\gamma$ ), the index $q=0$ (see equation [15]). In this case, $\mathcal{C}_{0}=1=\mathcal{C}_{1}$ and the equation of 
motion which defines the pressure has the obvious solution $p=\alpha^{\Gamma}$. In the general case, the index $q$ is given by

$$
q=\frac{2(\Gamma-\gamma)}{3 \Gamma-4}
$$

\subsection{Hydrostatic Equilibrium and Overdensity}

One useful reference state is that in which the core is in hydrostatic equilibrium at $t=0$ when the collapse begins. In the limit of large values of $x$, we thus expect the core to have no velocity so that $v=0$. In this limit, $x \gg v$, the equations of motion have solutions of the form

$$
\begin{gathered}
m(x)=\frac{2-\Gamma}{4-3 \Gamma}\left[\frac{2 \Gamma(4-3 \Gamma)}{(2-\Gamma)^{2}}\right]^{1 /(2-\Gamma)} x^{(4-3 \Gamma) /(2-\Gamma)}, \\
\alpha(x)=\left[\frac{2 \Gamma(4-3 \Gamma)}{(2-\Gamma)^{2}}\right]^{1 /(2-\Gamma)} x^{-2 /(2-\Gamma)} .
\end{gathered}
$$

The density profile of the hydrostatic equilibrium configuration is thus a power-law with index $\tilde{\mu} \equiv-2 /(2-\Gamma)$. Notice that the static equation of state alone determines the initial configuration (as expected). Notice also that solutions of this form make sense only for static equations of state with $\Gamma<4 / 3$. This latter result is, of course, well known from stellar structure theory (e.g., Chandrasekhar 1939).

In this paper, we allow for the initial states to have densities (and mass profiles) that are heavier than that appropriate for hydrostatic equilibrium. For general (static) equations of state, we let the parameter $\Lambda$ denote the overdensity, so that the initial mass and density profiles are given by the above equations with a leading factor of $\Lambda \geq 1$. For an isothermal (static) equation of state, however, we follow previous authors (e.g., Shu 1977) and write $\rho(r)=\mathcal{A} a_{s}^{2} / 2 \pi G r^{2}$ so that the starting profiles have the known forms $\alpha(x)=\mathcal{A} / x^{2}$ and $m(x)=\mathcal{A} x$. In this case, $\mathcal{A}=2$ corresponds to hydrostatic equilibrium and $\mathcal{A}>2$ represents overdense initial states. Note that $\Lambda=\mathcal{A} / 2$ for isothermal initial configurations.

It is useful to consider the density and mass profiles in terms of physical quantities. Including the constants that carry dimensions and the overdensity parameter, we can write the profiles in the form

$$
M(r)=4 \pi \Lambda \frac{2-\Gamma}{4-3 \Gamma}\left[\frac{\kappa \Gamma}{2 \pi G} \frac{4-3 \Gamma}{(2-\Gamma)^{2}}\right]^{1 /(2-\Gamma)} r^{(4-3 \Gamma) /(2-\Gamma)},
$$

and

$$
\rho(r)=\Lambda\left[\frac{\kappa \Gamma}{2 \pi G} \frac{4-3 \Gamma}{(2-\Gamma)^{2}}\right]^{1 /(2-\Gamma)} r^{-2 /(2-\Gamma)} .
$$




\subsection{Location of the Expansion Wave}

We can derive a general expression for the location of the expansion wave. If the initial state of the cloud core is in hydrostatic equilibrium, then the boundary between the inner collapsing region

and the outer static region will be a critical point in the flow. The mathematical manifestation of this critical point is that the matrix of coefficients in the system of differential equations $(9-13)$ must vanish (at the critical point). This condition can be written in the general form

$$
\gamma=\alpha^{1-\Gamma}(2-\Gamma)^{2} x_{H}^{2}
$$

where we have used the fact that $v=0$ and $p=\alpha^{\Gamma}$ at the head of the expansion wave $x_{H}$. Using the solutions for the starting density profile derived above, we can eliminate the reduced density $\alpha$ and find the following expression for the location $x_{H}$ of the head of the expansion wave

$$
x_{H}=\gamma^{(1-\Gamma / 2)}(2-\Gamma)^{-1}[2 \Gamma(4-3 \Gamma)]^{-(1-\Gamma) / 2} .
$$

Notice that this expression is valid for initial states that are in hydrostatic equilibrium $(\Lambda=1)$ and does not include the terms appropriate for nonzero initial velocities. In our analytic derivations, we use this expression as a useful benchmark for estimating infall rates. For future reference, it is also useful to have the (total) mass $m_{H}$ enclosed within the expansion wave. Using the starting density profile and the expression for $x_{H}$, we find

$$
m_{H}=2 \Gamma \gamma^{(2-3 \Gamma / 2)}[2 \Gamma(4-3 \Gamma)]^{-3(1-\Gamma) / 2}(2-\Gamma)^{-2}
$$

\subsection{Time Dependence of the Mass Infall Rate}

The static equation of state also determines the time dependence of the mass infall rate $\dot{M}$. The infall rate determines the time scales for the star formation processes. In addition, since these cores have no well defined mass scales, the mass infall rate is the physical quantity of importance for star formation. Using the above results, we find that the mass infall rate has the form

$$
\dot{M}=m_{0} \kappa^{3 / 2}(4-3 \Gamma)(4 \pi G)^{3(1-\Gamma) / 2} G^{-1} t^{3(1-\Gamma)},
$$

where $m_{0}$ is a constant which represents the reduced mass $m(x)$ in the inner limit $x \rightarrow 0$. Since the infalling material always approaches ballistic conditions in this inner limit, the magnitude of the reduced mass $m_{0}$ determines the infall rate. Notice that, except for this parameter $m_{0}$, which is expected to be roughly of order unity, the mass infall rate is completely specified by our scaling transformation. The remainder of this paper is (mostly) devoted to the determination of $m_{0}$ for the cases of interest.

For the case of an isothermal initial state, $\Gamma=1$, and the infall rate $\dot{M}$ is constant in time. Notice that for equations of state softer than the isothermal case $(\Gamma<1)$, the mass infall rate increases with time. On the other hand, for harder equations of state $(\Gamma>1)$, the mass infall rate 
decreases with time. Since we expect most star forming regions to have initial core configurations corresponding to soft equations of state, the general trend is for mass infall rates to be increasing functions of time.

\subsection{Matching onto Inner Solutions: Effects of Rotation}

In this section, we determine the most important effects of rotation on the collapse solutions. For the cases of interest, the inner limit of the spherical collapse flow approaches a ballistic (pressurefree) form. However, the spherical approximation must break down in the inner region of the flow where conservation of angular momentum plays an important role and where a circumstellar disk forms. We thus consider the inner limit of our spherical solutions as the outer limit of the rotating non-spherical solutions which we calculate below. This exercise in intermediate asymptotic analysis remains valid under the following ordering of (radial) size scales:

$$
R_{*} \ll R_{C} \ll r_{H}
$$

In this ordering constraint, the scale $R_{*}$ is the radius of the forming star and defines the inner boundary of the collapse flow. The scale $R_{C}$ is the centrifugal radius (defined more precisely below) which roughly divides the spherical outer region of the flow from the highly nonspherical inner region. Finally, the scale $r_{H}$ is the head of the expansion wave and (again roughly) divides the outer core from the collapsing inner core. This latter division is clean only for hydrostatic initial states.

Here, we present the form of the density profile resulting from the collapse of slowly rotating cloud cores. This calculation generalizes the isothermal case studied previously (Terebey, Shu, \& Cassen 1984), where the collapse solution in the outer portion of the core matches smoothly onto an "inner solution" that can be described analytically (Cassen \& Moosman 1981 and Ulrich 1976). In this inner regime, parcels of gas spiral inward on nearly ballistic trajectories with nearly zero total energy (and conserved specific angular momentum). The resulting orbits are parabolic and are described by the equation

$$
\frac{\mu_{0}\left(1-\mu_{0}^{2}\right)}{\mu_{0}-\mu}=\frac{1}{\zeta}
$$

where $\mu_{0}$ is the cosine of the angle $\theta_{0}$ of the asymptotically radial streamline (i.e., the parabolic fluid trajectory that is currently passing through the position given by $\zeta$ and $\mu \equiv \cos \theta$ initially made the angle $\theta_{0}$ with respect to the rotation axis). Although equation (33) is cubic in $\mu_{0}$ and has three roots, only one solution has physical significance. The quantity $\zeta$ is defined by

$$
\zeta \equiv \frac{j_{\infty}^{2}}{G M r}=\frac{R_{C}}{r}
$$

where $j_{\infty}$ is the specific angular momentum of parcels of gas currently arriving at the origin along the equatorial plane. We have followed previous authors in assuming an initial state that is rotating 
at a constant rotation rate $\Omega$, so that the quantity $j_{\infty}$ is given by

$$
j_{\infty}=\Omega r_{\infty}^{2}
$$

where $r_{\infty}$ is the starting radius of the material that is arriving at the origin at a given time. This radius can be determined by inverting the initial mass distribution $M(r)$, i.e.,

$$
r_{\infty}(M)=\left[\frac{M}{4 \pi \Lambda}\right]^{(2-\Gamma) /(4-3 \Gamma)}\left[\frac{2 \pi G}{\kappa \Gamma}(2-\Gamma)^{\Gamma}(4-3 \Gamma)^{1-\Gamma}\right]^{1 /(4-3 \Gamma)} .
$$

Putting all of these results together, we can find the centrifugal radius for any given static equation of state. In general form, the centrifugal radius can be written

$$
R_{C}=\Omega^{2}\left[\Lambda^{-4(2-\Gamma)} M^{4-\Gamma} G^{3(\Gamma-1)}(2 \kappa \Gamma)^{-1}(4-3 \Gamma)^{7-4 \Gamma}(2-\Gamma)^{4 \Gamma-6}(4 \pi)^{4 \Gamma-7}\right]^{1 /(4-3 \Gamma)},
$$

where the parameter $\Lambda \geq 1$ is the factor by which the initial states are denser than that required for hydrostatic equilibrium. The result for three specific cases are given below. For isothermal equations of state, $\Gamma=1$, we obtain the familiar result

$$
R_{C}=\frac{\Omega^{2} G^{3} M^{3}}{16 a_{s}^{8}} \sim t^{3}
$$

in the logatropic limit

$$
R_{C}=\frac{\Omega^{2} M}{2 \pi P_{0}} \sim t^{4}
$$

where $P_{0}$ is the coefficient in the equation of state (which has units of pressure in this limit).

Using equation (33) in conjunction with conservation of angular momentum and conservation of energy one can determine the velocity field (Cassen \& Moosman 1981). The density distribution of the infalling material can be obtained by applying conservation of mass along a streamtube (Terebey, Shu, \& Cassen 1984; Chevalier 1983), i.e.,

$$
\rho(r, \theta) v_{r} r^{2} \sin \theta d \theta d \phi=-\frac{\dot{M}}{4 \pi} \sin \theta_{0} d \theta_{0} d \phi
$$

Combining the above equations allows us to write the density profile in the form

$$
\rho(r, \theta)=\frac{\dot{M}}{4 \pi(G M)^{1 / 2}} r^{-3 / 2}\left(1+\frac{\mu}{\mu_{0}}\right)^{-1 / 2}\left[1+2 \zeta P_{2}\left(\mu_{0}\right)\right]^{-1},
$$

where $P_{2}\left(\mu_{0}\right)=\left(3 \mu_{0}^{2}-1\right) / 2$ is the Legendre polynomial of order two. Notice that this form for the density distribution is general in that it applies to all of the collapse calculations considered in this paper. The properties of the collapsing core determine the form of the functions $\dot{M}$ and $\zeta=R_{C} / r$ which appear in the general form (40). 


\section{COLLAPSE SOLUTIONS FROM ISOTHERMAL INITIAL STATES}

The solution for the collapse of a singular isothermal sphere has been found previously (Shu 1977) and has been used in numerous applications of star formation theory. Most applications use the "expansion-wave collapse solution" which starts from a hydrostatic initial state and has zero initial velocity. To account for the observed inward flows in starless cores, one can generalize the solution to include overdense initial states (as considered in Shu 1977) and/or nonzero starting velocities. In this section, we include both of these complications and show the relationship between them. We also generalize the collapse solution to include the effects of a dynamic equation of state which differs from isothermal, i.e., we set $\Gamma=1$ but allow $\gamma \neq 1$. For these solutions, the parameter $\kappa$ that appears in the static equation of state has units of speed squared and can be written in terms of the isothermal sound speed, i.e., $\kappa=a_{s}^{2}$.

\subsection{Self-similar Solutions}

For the case of an isothermal initial state, with static $\Gamma=1$, the parameter $q=2(\gamma-1)$ and the reduced equations of motion take the form

$$
\begin{gathered}
(v-x) \frac{d v}{d x}+\frac{1}{\alpha} \frac{d p}{d x}=-\frac{m}{x^{2}}, \\
(v-x) \frac{1}{\alpha} \frac{d \alpha}{d x}+\frac{d v}{d x}=2(1-v / x),
\end{gathered}
$$

where the reduced pressure $p(x)$ and mass $m(x)$ are given by

$$
p=\mathcal{C} \alpha^{\gamma} m^{2(\gamma-1)} \quad \text { and } \quad m=x^{2} \alpha(x-v) .
$$

In this paper, we explicitly consider the starting states to exhibit two generalizations from exact hydrostatic equilibrium. The cores can be overdense, i.e., with densities larger than can be pressure supported so that $\mathcal{A}>2$. We also consider the possibility of nonzero initial flows as characterized by the initial speed $v_{\infty}$. As a result, we take the starting states to have the form

$$
\alpha=\frac{\mathcal{A}}{x^{2}}\left[1-\frac{\mathcal{A}-2}{2 x^{2}}+\mathcal{O}\left(x^{-4}\right)\right]
$$

and

$$
v=-v_{\infty}-\frac{\mathcal{A}-2}{x}-\frac{v_{\infty}}{x^{2}}-\frac{(\mathcal{A}-2)[(5-2 \gamma) / 3-\mathcal{A} / 6]-2 v_{\infty}^{2}}{x^{3}}+\mathcal{O}\left(x^{-4}\right) .
$$

The density profile has the same form as considered previously for overdense states (Shu 1977), whereas the velocity field picks up additional terms due to $v_{\infty} \neq 0$ and $\gamma \neq 1$. The limit of hydrostatic equilibrium corresponds to $\mathcal{A} \rightarrow 2$ and $v_{\infty} \rightarrow 0$, so that $v \rightarrow 0$ and $\alpha \rightarrow 2 / x^{2}$.

For isothermal starting states (static $\Gamma=1$ ), we are left with a three parameter family of collapse solutions, as specified by the density parameter $\mathcal{A}$, the inward velocity scale $v_{\infty}$, and the 
index $\gamma$ of the dynamic equation of state. For each choice $\left(\mathcal{A}, v_{\infty}, \gamma\right)$, we get a different similarity solution, and, in particular, a value of the reduced mass at the origin $m_{0}$ (which specifies the mass infall rate). The standard infall-collapse solution (Shu 1977) has $m_{0}=0.975$ and corresponds to a particular point in this parameter space, namely $(2,0,1)$; that same paper also presents solutions for points $(\mathcal{A}, 0,1)$, although they are not widely used in subsequent work.

The first results of this paper are summarized by Figures 1 and 2. In Figure 1, we plot the reduced mass $m_{0}$ as a function of the overdensity parameter $\mathcal{A}$ for various values of the index $\gamma$ appearing in the dynamic equation of state. Figure 1 shows that the dynamic equation of state has only a modest effect on the mass infall rate, whereas variations in the overdensity $\mathcal{A}$ are much more important. Similarly, Figure 2 shows the variation of the reduced mass $m_{0}$ as the initial inward velocity $v_{\infty}$ increases. In this case, the dynamic equation of state affects the $m_{0}$ values more than in the case of overdense states, but variation of the inward speed $v_{\infty}$ has greater influence.

\subsection{Numerical Collapse Solutions}

The numerical treatment of the collapse problem can be used to verify the semi-analytic results and to study alternate collapse scenarios that are not fully self-similar. For this numerical exploration, we specialize to the case $\Gamma=1=\gamma$. We consider collapse solutions for various values of the overdensity parameter $\mathcal{A}$, where $\mathcal{A}=2$ is the value appropriate for hydrostatic equilibrium (where we adopt the notation of Shu 1977 for isothermal starting states). In addition, we allow for a non-zero infall velocity $u_{\text {in }}$ in the initial state. As discussed previously, our self-similar treatment considers nonzero velocities in the outer region with the specific profile given by equation (46) for isothermal initial conditions. We note that current observations measure the inward velocities at a limited range of local radii, so a variety of different velocity fields (inward speed as a function of radius) remain possible. Here, we numerically determine the infall rates for a collection of isothermal spheres with nonzero outer velocities that are constant (with radius). The speed $u_{\text {in }}$ and the overdensity parameter $\mathcal{A}$ are thus the two parameters that can be varied.

Figure 3 shows one representative model with $\mathcal{A}=2.0$ (a density profile in hydrostatic equilibrium) and initial inward speed $u_{i n}=a_{s} / 2$ (a typical observed value). The top panel of Figure 3 shows the density profile at the initial time (dotted curve) and four subsequent times. Except for the overall normalization constant, this solution is nearly identical to that found in the original self-similar collapse solutions (compare with Figure 3 of Shu 1977). The second panel shows the velocity profile. The inner regime approaches the ballistic (free-fall) form, $|v| \sim r^{-1 / 2}$, as expected. On the outside (large radii), the inward velocity attaches smoothly onto the imposed boundary condition $v=$ constant. The numerically calculated infall rate quickly approaches the constant value predicted by the similarity solution and stays fixed at that value.

Figure 4 illustrates that the flow is indeed self-similar (as expected). Here, the collapse solutions for both the density (top panel) and velocity field (bottom panel) at four different times are rescaled 
according to the similarity transformation of $\S 2$ and then plotted together. The result is one smooth function for both density and velocity, as expected for self-similar flow fields.

The resulting values of $m_{0}$ are shown in Figure 5 for a set of models with varying overdensities (specified by $\mathcal{A}$ ) and varying infall speeds in the range $v_{\infty}=u_{i n} / a_{s}=0-1.0$. Figure 5 shows that the mass infall rates (specified through $m_{0}$ ) increase with increasing overdensity $\mathcal{A}$ and with increasing initial inward speed $v_{\infty}$. Furthermore, the functional dependence of $m_{0}$ on both $\mathcal{A}$ and $v_{\infty}$ is approximately linear so that increasing $\mathcal{A}$ and increasing $v_{\infty}$ produce similar effects on the collapse solutions. A more detailed comparison of the effects of $\mathcal{A}$ and $v_{\infty}$ on the mass infall rate emerges from the analytical estimates derived in the following subsection.

\subsection{Analytic Estimates}

In this subsection, we derive analytic estimates for $m_{0}$ for the case of initial configurations with static $\Gamma=1$. We consider, separately, both the case of overdense initial states with $\mathcal{A}>2$ and initial states with nonzero inward velocities $v_{\infty}$ at the start. To start, for simplicity, we set dynamic $\gamma=1$. These estimates, by necessity, require approximations, but in the end they provide both analytic understanding of the collapse physics and accurate analytic formulas for the integration constants $m_{0}$ and hence the infall rates.

We first consider the case of overdense initial states. For a given fluid layer at initial radius $r_{0}$, the inward velocity is zero at $t=0$ (where $x \rightarrow \infty$ ), but an inward flow soon develops. Due to the self-similar nature of the problem, the value of the radius $r_{0}$ does not matter. To estimate $m_{0}$, we assume here that the fluid layer migrates slowly inward as a nonzero inward velocity develops. As the layer moves inward, the expansion wave moves outward. When the expansion wave passes

the fluid layer on its way out, we assume that the layer subsequently falls inward in a pressure-free manner. For a starting radius $r_{0}$, the fluid layer has a radial location at later times given by

$$
r^{2}=r_{0}^{2}-(\mathcal{A}-2) a_{s}^{2} t^{2}
$$

where $a_{s}$ is the sound speed of the initial state. The expansion wave has radial location $r_{H}=a_{s} t$, so the expansion wave crosses the fluid layer at time $t_{c}$ given by

$$
t_{c}=\left(r_{0} / a_{s}\right)(\mathcal{A}-1)^{-1 / 2}
$$

At this time, the layer resides at radius $r_{c}$ given by

$$
r_{c}=r_{H}=a_{s} t_{c}=r_{0}(\mathcal{A}-1)^{-1 / 2} \text {. }
$$

From this location at $r_{c}$, the layer collapses in a pressure-free manner. The layer is already moving inward with speed

$$
u_{\text {inc }}=\frac{a_{s}^{2}(\mathcal{A}-2)}{r_{H}} t_{c}=a_{s}(\mathcal{A}-2)
$$


where we have kept only the leading order term in the expansion. With this starting speed, the time required for the fluid layer to fall to the origin is given by

$$
t_{f}=r_{c}^{3 / 2}\left[2 G M\left(r_{0}\right)\right]^{-1 / 2} f(\eta)
$$

where we have defined

$$
f(\eta) \equiv(1-\eta)^{-3 / 2}\left\{\sin ^{-1}(1-\eta)^{1 / 2}-\left(\eta-\eta^{2}\right)^{1 / 2}\right\}
$$

where the parameter $\eta \equiv u_{i n c}^{2} r_{c} /\left[2 G M\left(r_{0}\right)\right]$ measures the size of the initial velocity. The effect of $u_{\text {inc }}$ is thus determined by the dimensionless function $f(\eta)$. Here, the enclosed mass is $M\left(r_{0}\right)$, i.e., all of the mass within the starting radius $r_{0}$, even though the layer now resides at the smaller radius $r_{c}$. As result, the relevant value of $\eta$ is given by

$$
\eta=\frac{(\mathcal{A}-2)^{2}}{(2 \mathcal{A})(\mathcal{A}-1)^{1 / 2}} .
$$

In the limit of hydrostatic equilibrium, $\mathcal{A} \rightarrow 2$ and $\eta \rightarrow 0$, so that we recover the classical result $f(\eta) \rightarrow \pi / 2$. Since the inward speed cannot be larger than the free-fall value, this formula is only valid for $\mathcal{A} \leq \mathcal{A}_{\max } \approx 9.35$ (although we are interested in more modest values of $\mathcal{A}$ ). The infall time can be written

$$
t_{f}=f(\eta) \frac{r_{0} / a_{s}}{(\mathcal{A}-1)^{3 / 4}(2 \mathcal{A})^{1 / 2}} .
$$

The total time for the fluid layer to fall is then given by the sum $t_{T}=t_{c}+t_{f}$, i.e.,

$$
t_{T}=\frac{r_{0} / a_{s}}{(\mathcal{A}-1)^{1 / 2}}+\frac{f(\eta) r_{0} / a_{s}}{(\mathcal{A}-1)^{3 / 4}(2 \mathcal{A})^{1 / 2}} .
$$

By the time $t_{T}$ that the fluid layer reaches the origin, the expansion wave has traveled out to $r_{H}=a_{s} t_{T}$. After some algebra, the ratio of reduced masses can be written in the form

$$
\frac{m_{0}}{m_{H}}=\left\{(\mathcal{A}-1)^{-1 / 2}+f(\eta)(2 \mathcal{A})^{-1 / 2}(\mathcal{A}-1)^{-3 / 4}\right\}^{-1} .
$$

Since $m_{H}=\mathcal{A}$, this estimate for $m_{0}$ is now completely specified. Notice that for sufficiently large value of $\mathcal{A}$ (namely, $\mathcal{A} \gtrsim 2.85$ ), the ratio $m_{0} / m_{H}$ can exceed unity. This occurs because the inward velocities outside the nominal location of the expansion wave move material inward faster than the outward-moving expansion wave can enclose more material.

Figure 6 shows a comparison of our self-similar formulation, the analytic estimate derived above, and results from our numerical treatment. For this comparison, all initial states are taken to be isothermal (so that static $\Gamma=1$ ) and the collapse flow is assumed to be isothermal (so that dynamic $\gamma=1$ ). The resulting values of the dimensionless mass $m_{0}$, which specifies the mass infall rate, are shown as a function of the overdensity parameter $\mathcal{A}$. Notice that all of the curves are in good agreement. The self-similar results (solid curve) have been determined previously (see Table I of Shu 1977), but such overdense solutions have been largely ignored. The numerical results (dotted 
curve) demonstrate that the self-similar formulation produces the correct infall rates for cores that start with the same initial conditions (here, density profiles that are sufficiently centrally condensed - see also §4.4). In addition, the analytic estimates are in excellent agreement with both of the more rigorously determined values. The analytic treatment makes two approximations, which tend to cancel out: We first assume that once the expansion wave overtakes a fluid layer, the material immediately becomes ballistic, and hence this assumption acts to overestimate the infall rate. We also assume that the initial speed in given by equation (50), which keeps only the leading term in a series expansion, and hence tends to underestimate the infall rate. As a result, the dependence of $m_{0}$ on the overdensity $(\mathcal{A})$ is well described by equation $(56)$. In spite of its derivation, the result (eq. [56]) provides an accurate and user-friendly formula to specify infall rates.

We now present an analogous calculation for the case of initial inward velocities $\left(u_{i n} \neq 0\right)$. To isolate the effects of the velocity, we fix the density coefficient to be that appropriate for hydrostatic equilibrium so that $\mathcal{A}=2$. For a fluid layer at starting radius $r_{0}$, the inward speed is nearly constant at $u_{i n}$, and the radial location as a function of time is given by

$$
r(t)=r_{0}-u_{i n} t
$$

Using the standard expression for the expansion wave, which is only an approximation in the present context (see eq. [28]), we can find the time $t_{c}$ at which the expansion wave crosses the fluid layer,

$$
t_{c}=r_{0}\left(u_{i n}+a_{s}\right)^{-1}
$$

and the corresponding location $r_{c}$ of the layer,

$$
r_{c}=r_{0} a_{s}\left(u_{i n}+a_{s}\right)^{-1} .
$$

As before, we assume that the fluid layer collapses subsequently in a pressure-free manner. The additional time $t_{f}$ (beyond $t_{c}$ ) required for the fluid layer to reach the center is still given by equation (54), with the intermediate crossover radius $r_{c}$ given by equation (59) and the parameter $\eta$ given by

$$
\eta=\frac{v_{\infty}^{2}}{4\left(1+v_{\infty}\right)}
$$

Keep in mind that $v_{\infty}=u_{i n} / a_{s}$. Thus far, we have assumed that the fluid layer moves inward at constant speed $u_{\text {in }}$ until it crosses the expansion wave and then falls inward with no pressure forces. In actuality, the layer will accelerate a small amount before crossing the expansion wave, but will be held back (again, a small amount) by pressure forces; these two corrections thus tend to cancel. However, we can account for this complication by introducing a dimensionless parameter $b$ through the ansatz $\widetilde{v}_{\infty}=b v_{\infty}$. After some algebra, the ratio of reduced masses takes the form

$$
\frac{m_{0}}{m_{H}}=\left\{\left[\frac{1}{1+\widetilde{v}_{\infty}}\right]+\frac{1}{2} f\left[\eta\left(\widetilde{v}_{\infty}\right)\right]\left[\frac{1}{1+\widetilde{v}_{\infty}}\right]^{3 / 2}\right\}^{-1}
$$

The mass contained within the expansion wave $m_{H}=2$ for these configurations, so the mass $m_{0}$ is completely specified. 
Figure 7 shows how this analytic formula compares with the results of the self-similar formulation and the numerical treatment of the collapse problem. All of the initial states are taken to have $\mathcal{A}=2$, as well as $\Gamma=1=\gamma$. The resulting values of the reduced mass $m_{0}$ are shown as a function of the starting inward speed $v_{\infty}=u_{i n} / a_{s}$. The solid curve shows the results of the selfsimilar calculation and the dashed curve shows the results of the numerical treatment. The dotted curve shows the analytic estimate derived above, where we set $b=1.175$ to take into account the (small) acceleration of the fluid layer, relative to the simple assumptions of the derivation. With this correction, the analytic estimate is in good agreement with both the numerical and self-similar results.

These analytic derivations allow us to make an approximate transformation between the effects of increasing the initial density $(\mathcal{A}>2)$ and increasing the starting inward velocity $\left(v_{\infty}>0\right)$. If we equate the derived expressions for $m_{0}$ for the two cases (eqs. [56] and [61]), we find that to a reasonable approximation $\mathcal{A}$ and $v_{\infty}$ are related through the cubic equation

$$
\mathcal{A}^{2}(\mathcal{A}-1) \approx 4\left(1+v_{\infty}\right)^{2}
$$

For a typically observed value of $v_{\infty}=0.5$, for example, the corresponding value of $\mathcal{A}$ that produces the same infall rate is $\mathcal{A} \approx 2.5$. For this case, $m_{0} \approx 2.1$ and the infall rate is larger than that of the infall-collapse solution (Shu 1977) by 115 percent (a factor of 2.15); this result agrees with a recent calculation for the collapse of a magnetized singular isothermal toroid (Allen et al. 2003b). Observations indicate that inward motions span a range $0.04-0.1 \mathrm{~km} / \mathrm{s}$ (Lee et al. 1998), which correspond to either starting speeds in the range $v_{\infty}=u_{i n} / a_{s}=0.1-0.5$ or overdensities in the range $\mathcal{A}=2.1-2.5$. The largest value of $v_{\infty}$ that one should consider is $v_{\infty}=1$, which limits the overdensity parameter to the range $\mathcal{A} \leq 2.9$ (using eq. [62]).

\subsection{Relation to Observations}

Given the three parameter family of collapse solutions found thus far, we need to specify the portion of the parameter space $\left(\mathcal{A}, v_{\infty}, \gamma\right)$ that corresponds to observed molecular cloud cores. Since the dynamic equation of state (specified by $\gamma$ ) does not affect the mass infall rates as much as the other variables (Figures $1-7$ ), we focus this discussion on cases with $\gamma=1=\Gamma$. This paper is motivated by the realization that some starless cores are observed to have subsonic inward motions (Lee et al. 1999, 2001) at roughly half the sound speed, i.e., $u_{i n} \approx a_{s} / 2$. As mentioned previously, these observed motions could arise in two different ways: (1) The inward speed could be part of the initial conditions; in this case, we model the collapse using a starting velocity $v_{\infty} \neq 0$. (2) The observed speeds could arise because the core has already begun to collapse on the outside; in this case, we model the collapse using overdense initial states $(\mathcal{A}>2)$, which have zero velocity at $t=0$ but soon develop subsonic speeds in the outer regions (beyond the expansion wave). We show here that both of these possibilities can be made consistent with current observations, although the $v_{\infty} \neq 0$ case is favored. 
Both scenarios $\left(\mathcal{A}>2\right.$ or $\left.v_{\infty}>0\right)$ are constrained by the observation that the cores in question exhibit inward motions but do not contain detectable infrared sources. The theory is constrained because the observed inward velocity must be large enough and the predicted protostellar luminsoity must be small enough. For overdense initial states, the predicted inward speed $u_{i n}$ at a given radius $r_{o b s}$ of observation is given by

$$
u_{\text {in }}=(\mathcal{A}-2) a_{s}^{2} t / r_{o b s}
$$

where $t$ is the time since the onset of collapse. The luminosity $L_{t h}$ of the central source can be written in the form

$$
L_{t h}=\mathcal{F} \frac{G M \dot{M}}{R_{*}}=\mathcal{F} m_{0}^{2} \frac{a_{s}^{6}}{G R_{*}} t
$$

where $\mathcal{F}$ is the fraction of the total available luminosity $L_{0}$ (see eq. [1]) that is realized in the central source. To match the observations, the predicted inward speeds must be as large as those seen, $u_{i n} \geq u_{o b s}$. At the same time, the predicted luminosity must be less than the observed limit, $L_{t h} \leq L_{\text {lim }}$ (where $L_{\text {lim }}=0.1-1 L_{\odot}$ ). The first of these constraints implies a lower limit on the time $t$ since collapse began, while the second constraint implies an upper limit on $t$. In order to satisfy both constraints, the following bound must be met

$$
\mathcal{F} \frac{u_{i n}}{a_{s}} \leq L_{l i m} \frac{R_{*} G}{r_{o b s} a_{s}^{5}} \frac{\mathcal{A}-2}{m_{0}^{2}} \approx 0.05 \frac{\mathcal{A}-2}{m_{0}^{2}} \widetilde{L}
$$

where $\widetilde{L} \equiv L_{l i m} /\left(1 L_{\odot}\right)$, and the numerical constant on the right hand side is obtained by assuming $r_{\text {obs }}=0.1 \mathrm{pc}, a_{s}=0.2 \mathrm{~km} / \mathrm{s}$, and $R_{*}=2 \times 10^{11} \mathrm{~cm}$ (Stahler et al. 1981). The function $F(\mathcal{A}) \equiv$ $(\mathcal{A}-2) / m_{0}^{2}$, considered as a function of $\mathcal{A}$, has a maximum value of about 0.112 at $\mathcal{A} \approx 2.5$ (found by evaluation), and we obtain the constraint

$$
\mathcal{F} \frac{u_{i n}}{a_{s}} \leq 0.006 \widetilde{L}
$$

The observed inward velocities lie in the range $u_{o b s} / a_{s}=0.25-0.5$, so this constraint requires the luminosity to be a small fraction of the total available luminosity, namely $\mathcal{F} \lesssim 0.02$. This tight constraint greatly limits the available parameter space for the case in which the observed inward velocities are part of the collapse $(\mathcal{A}>2)$.

For the scenario in which the inward velocities are part of the initial conditions, the observation that $v_{\infty}=u_{i n} / a_{s}=0.25-0.5$ is built into the boundary conditions and does not further constrain the solution. However, the requirement that the core does not display a detectable infrared source implies a time constraint,

$$
\mathcal{F} t \leq L_{l i m} \frac{R_{*} G}{a_{s}^{6} m_{0}^{2}} \approx 1.3 \times 10^{4} \operatorname{yr}\left(\widetilde{L} / m_{0}^{2}\right)
$$

where we have used the same numerical values as before. In order to account for $u_{\text {obs }} / a_{s}=0.25$ $-0.5=v_{\infty}$, the implied values of $m_{0}$ lie in the range $m_{0}=1.6-2.2$. As a result, we obtain a constraint of the form $\mathcal{F} t \lesssim 5100 \mathrm{yr}$. In order to allow for a respectably long viewing time, say $t \sim 5 \times 10^{4}$ yr (about half the expected collapse time), we would need $\mathcal{F} \lesssim 0.10$. 
Although both scenarios can be made consistent with observations, the case of overdense initial states is much more constrained. This overdense scenario - in which the observed inward motions are part of the collapse itself - requires extremely inefficient power generation in the central source, $\mathcal{F} \lesssim 0.02$. These solutions are also constrained in that they cannot be extended to $t<0$ to become "complete" solutions (again, see Hunter 1977). In constrast, the $v_{\infty} \neq 0$ scenario - in which the observed inward motions are part of the initial conditions - is less constrained, but still requires $\mathcal{F} \lesssim 0.10$ under reasonable assumptions.

\section{COLLAPSE SOLUTIONS FROM NON-ISOTHERMAL INITIAL STATES}

In this section, we consider the collapse of molecular cloud cores with initial density profiles that are shallower than $\rho \propto r^{-2}$ (the profile of the singular isothermal sphere). As discussed in previous papers (e.g., Lizano \& Shu 1989, McLaughlin \& Pudritz 1997), these shallower density profiles arise from hydrostatic equilibrium models of gaseous spheres with equations of state that are softer than isothermal. In the present context, such states correspond to static $\Gamma<1$. The selfsimilar collapse of such spheres have been considered previously for the case of $\gamma=\Gamma$ (see Cheng 1977, 1978, and the appendices to McLaughlin \& Pudritz 1997), although the focus has been on hydrostatic initial conditions with $v_{\infty}=0$. In this section, we generalize the self-similar collapse problem to include nonisothermal states that are either overdense $(\Lambda>1)$ or contain nonzero inward velocities $\left(v_{\infty}>0\right)$. Notice also that the motivation for considering equations of state that are softer than isothermal applies primarily to the static equation of state, and not necessarily to the dynamic equation of state. We thus generalize these collapse solutions to include the case $\gamma \neq \Gamma$.

\subsection{Self-similar Collapse Solutions with a Single Equation of State}

We first consider the case where $\gamma=\Gamma$ and generalize previous work to include non-zero velocities. As discussed earlier, the observed inward motions can arise in two conceptually different ways: Such velocities can be "part of the collapse" and hence would imply overdense initial states with $\Lambda>1$; the velocities could also arise as "part of the initial conditions" and would imply $\Lambda=1$

and $v_{\infty}>0$. In either case, we can obtain self-similar solutions using standard methods. The equation of motion reduce to the form

$$
\begin{aligned}
y \frac{d v}{d x}+\Gamma \alpha^{\Gamma-2} \frac{d \alpha}{d x} & =\frac{y \alpha}{4-3 \Gamma}+(\Gamma-1) v, \\
\frac{d v}{d x}+\frac{y}{\alpha} \frac{d \alpha}{d x} & =2(1-v / x),
\end{aligned}
$$

where we have defined $y \equiv(\Gamma-2) x+v$. In order to consider overdense states and nonzero initial inward motions, we must specify the outer boundary conditions. Keep in mind that the limit $x \rightarrow \infty$ 
corresponds to either $r \rightarrow \infty$ or $t \rightarrow 0$ because of the nature of the similarity transformation. The starting states are thus a generalization of the results given in $\S 4.1$ and take the form

$$
\begin{gathered}
\alpha=\Lambda \beta_{0} x^{-2 /(2-\Gamma)}\left\{1-\Delta_{0} \frac{3 \Gamma-2}{2(2-\Gamma)} x^{-2 /(2-\Gamma)}\right\}, \\
v=-v_{\infty}-\Delta_{0} x^{-\Gamma /(2-\Gamma)}
\end{gathered}
$$

where we have defined the dimensionless coefficients

$$
\beta_{0}=\left[\frac{2 \Gamma(4-3 \Gamma)}{(2-\Gamma)^{2}}\right]^{1 /(2-\Gamma)} \quad \text { and } \quad \Delta_{0}=\Lambda \beta_{0} \frac{2-\Gamma}{4-3 \Gamma}\left[1-\Lambda^{-(2-\Gamma)}\right]
$$

We note that the equations of motion can contain critical points, which can be considered as generalized sonic points. The condition for the flow to pass through a critical point take the form

$$
[(\Gamma-2) x+v]^{2}=\Gamma \alpha^{\Gamma-1}
$$

Although the fluid fields are continuous at the critical points (defined by eq. [73]), the derivatives (here, with respect to the similarity variable $x$ ) of the fluid fields need not be. As a result, care must be taken when numerically integrating through critical points. One can expand the functions in the neighborhood of the critical points and use the resulting analytic forms to integrate through the point. This treatment has been discussed previously in the literature (e.g., see Shu 1977 and McLaughlin \& Pudritz 1997) and need not be belabored here. In the present application, only solutions starting from hydrostatic initial conditions actually go through the critical points. For starting states that are even slightly overdense, or ones that start with small initial inward velocities, the solutions avoid the critical points altogether.

The basic results of this section are shown in Figure 8 and 9. Keep in mind that the infall rate, the quantity of interest in this collapse calculation, is given by the analytic expression of equation (31), with the reduced mass $m_{0}$ determined numerically (as shown in the Figures). Figure 8 shows the reduced mass $m_{0}$ as a function of the index $\Gamma=\gamma$ for varying values of the overdensity parameter $\Lambda$. The results are plotted as the ratio $m_{0} / m_{H}$, where $m_{H}$ is the mass enclosed within the expansion wave for the case of hydrostatic equilibrium, i.e., with $\Lambda=1$. During collapse, the overdense states develop a rapid inward flow well beyond the nominal location of the expansion wave (which is most meaningful for the hydrostatic case). As a result, the mass infall rates become rather large, and vary with overdensity more sensitively than in the case of the isothermal collapse calculations of the previous section. Specifically, as the density increases by a factor of $2(\Lambda=1 \rightarrow 2), m_{0}$ increases by a factor of $\sim 1840$ in the logatropic limit $\Gamma \rightarrow 0$, compared to only a factor of 5.7 in the isothermal limit $\Gamma \rightarrow 1$. Notice also that because of the rapid speed at large $r$, the ratio $m_{0} / m_{H}$ can be greater than unity.

Figure 9 shows the corresponding results for the reduced mass $m_{0}$ for varying values of the fixed inward speed $v_{\infty}$. As before, the results are presented in terms of the ratio $m_{0} / m_{H}$, where $m_{H}$ 
is the hydrostatic value (which is constant for all $v_{\infty}$ ). These solutions have a more complicated interpretation than their isothermal counterparts of the previous section. For isothermal initial conditions, the reduced speed $v$ is just the physical speed $u$ scaled by the (constant) isothermal sound speed. For nonisothermal conditions, the physical speed $u=w v(x)$, where $v$ is the (dimensionless) reduced velocity and $w \equiv \kappa^{1 / 2}\left(4 \pi G t^{2}\right)^{(1-\Gamma) / 2}$ is (esssentially) the effective sound speed at the head of the expansion wave. Unlike the isothermal case, however, the scaling speed $w$ is time dependent. Self-similar solutions can still be found (Figure 9), but the interpretation is not as clean as before.

\subsection{Estimate for $m_{0}$ for Hydrostatic Initial Conditions}

In this section, we derive an estimate for the reduced mass $m_{0}$ at the origin for the case of hydrostatic initial states. This estimate is found by calculating the value of $m_{0}$ that would arise for a pressure-free collapse. Since the pressure forces are small, this calculation should provide a reasonable estimate of the true value of $m_{0}$. The pressure forces are not zero, however, and retard the flow so that the true value of $m_{0}$ will be smaller than given by this estimate. We correct for this difference using a simple analytical fit.

To start, we consider a fluid layer at an initial radius $r_{0}$. Because of the self-similar nature of the problem, the value of $r_{0}$ does not matter. For the collapse solutions of interest, the fluid layer will remain at rest until the expansion wave reaches the radius $r_{0}$; afterward, the layer begins to fall. As the layer falls inward, the total mass enclosed within its current radial position remains constant and is given by the hydrostatic profile $(\S 3.2)$ evaluated at $r_{0}$, i.e., $M\left(r_{0}\right)$.

The time $t_{f}$ required for a fluid layer to fall from an initial radius $r_{0}$ is given by

$$
t_{f}=\frac{\pi}{2} r_{0}^{3 / 2}(2 G M)^{-1 / 2},
$$

where we have assumed no pressure forces so that this time scale is a lower limit. Notice that if the fluid layer is not assumed to start at rest, but rather has an initial inward velocity $v_{0}$, then the leading numerical coefficient will be different (not $\pi / 2$ ).

The time $t_{c}$ for the expansion wave to reach the initial radius $r_{0}$ is given by

$$
t_{c}=\left[A r_{0} / x_{H}\right]^{1 /(2-\Gamma)},
$$

where $A$ is the coefficient in the similarity transformation given by equation (22). When the fluid layer reaches the origin, the total time $t_{T}$ that has elapsed is given by the sum

$$
t_{T}=t_{c}+t_{f}
$$

The total mass in the central object (at the origin) is the mass enclosed within the radius $r_{0}$. The total mass enclosed with the expansion wave is given by the hydrostatic mass profile (see §3.2) evaluated at the location of the expansion wave radius at time $t_{T}$, i.e., at

$$
r_{H}=x_{H} A^{-1} t_{T}^{2-\Gamma} \text {. }
$$


Thus, the ratio of masses is given by

$$
\frac{m_{0}}{m_{H}}=\left[\frac{r_{0}}{r_{H}}\right]^{(4-3 \Gamma) /(2-\Gamma)}=\left[\frac{t_{0}}{t_{T}}\right]^{(4-3 \Gamma)} .
$$

In this case, since the initial states are hydrostatic, $r_{0}=r_{H}$ and the ratio $m_{0} / m_{H}$ is always less than unity (compare with the overdense states of $\S 4.3$ ).

We can use the similarity transformation and the hydrostatic initial state to write the pressurefree collapse time $t_{0}$ as a fraction of the total time $t_{T}$. We also introduce a correction factor $g>1$, which can be a function of the equation of state, and which accounts for the fact that the true infall time is longer than the pressureless free fall time calculated above. After some algebra, the mass ratio can be written in the form

$$
\frac{m_{0}}{m_{H}}=\left\{1+g \frac{\pi}{4}(\gamma / \Gamma)^{1 / 2}(2-\Gamma)^{-1 / 2}\right\}^{-(4-3 \Gamma)} .
$$

Since we already have a closed form expression for the enclosed mass $m_{H}$ (equation [30]), we thus have a completely analytic estimate for $m_{0}$. Also, since this estimate was derived assuming no pressure forces, this expression (with $g=1$ ) represents an upper limit for the reduced mass $m_{0}$. As an example, we consider the limit of the singular isothermal sphere (Shu 1977) where $\gamma=\Gamma=1$. For this case, $m_{H}=2$ and we obtain the estimate $m_{0}=8 /(4+\pi) \approx 1.12$, which compares reasonably

well with the calculated value of $m_{0}=0.975$. Although the expression (79) is only accurate at the 10 percent level, it illustrates how the mass infall rate (which is proportional to $m_{0}$ - equation [31]) varies with the equation of state (mostly the static index $\Gamma$ ).

Figure 10 shows the values of the central mass $m_{0}$ (which sets the mass infall rate) as a function of the index $\Gamma$ in the static equation of state for the simplest case in which the dynamic $\gamma$ is the same as the static $\Gamma$. The solid curve shows the results obtained from solving the self-similar equations of motion (this result has been obtained previously - see McLaughlin \& Pudritz 1997). The dashed curve shows our analytic approximation for $m_{0}$, where we take into account the fact that pressure slows down the infall so that the time required to fall from a given starting radius is longer by a factor of $g$ (see equation [79]). This effect is greater for softer equations of state, so we take $g=g(\Gamma)=(4 / 3)(2-\Gamma)^{9 / 10}$, where the index of $9 / 10$ is found by fitting the numerically determined curve. The result provides almost an exact fit.

\subsection{Different Dynamic and Static Equations of State}

Finally, we consider collapse flow with nonisothermal initial states and varying choices for the dynamic equation of state. Figure 11 shows the result of keeping a fixed static $\Gamma$ while allowing the dynamic equation of state (dynamic $\gamma$ ) to vary. As shown, the variation in the dynamic equation of state has relatively little effect on the resulting reduced masses $m_{0}$, except when the dynamic 
index $\gamma$ is much larger than the static index $\Gamma$. In this latter regime, the pressure forces within the incoming flow become large and the infall rates are suppressed. This suppression is best illustrated by considering a standard reference case where the static index $\Gamma$ is arbitrary, but the dynamic equation of state is chosen to be isothermal and fixed $(\gamma=1)$. Figure 12 shows the result for varying static indices $\Gamma$ and a fixed (isothermal) dynamic equation of state. The ratio $m_{0} / m_{H}$ of the central mass variable to the mass within the fiducial expansion wave (this ratio determines the mass infall rate) is plotted as a function of the static index $\Gamma$ (as before, the denominator uses the hydrostatic value of $m_{H}$ with $\Lambda=1$ ). The solid curves show the case of $\gamma=\Gamma$ for two choices of the overdensity $(\Lambda=1.5$ and 2$)$. The dashed curves show the resulting values of $m_{0} / m_{H}$ for the same starting conditions, and the same static $\Gamma$, but with dynamic $\gamma=1$. This generalization to

$\gamma \neq \Gamma$ leads to increased pressure forces during the collapse (relative to the case of $\Gamma=\gamma$ ) and the resulting values of $m_{0}$ are smaller. The discrepancy grows with decreasing static index $\Gamma$.

Before leaving this section, we note that the mathematical formulation of the collapse problem used in this paper $(\S 2)$ does not allow self-similar solutions for the case with $\Gamma \rightarrow 0$ and $\gamma \neq \Gamma$. In other words, in the logatropic limit, collapse with other dynamic equations of state remains undefined. One manifestation of this problem can be found in equation (16) for the reduced pressure. In the limit $\Gamma \rightarrow 0(\gamma \neq 0), p \rightarrow$ constant instead of the required limiting form $p=\log \alpha$. The class of solutions found in this paper are known mathematically as self-similar solutions of the first kind (Barenblatt 2003). Although the case of $\Gamma \rightarrow 0, \gamma \neq 0$ does not allow for a self-similar solution of the first kind, it remains possible that the problem will admit self-similar solutions of the second kind, also known as incomplete similarity (Barenblatt 2003; Zeldovich 1956; von Weizsäcker 1954). This complication is beyond the scope of this paper and will be left for future consideration.

\section{CONCLUSION}

\subsection{Summary of Results}

In this paper, we have explored a wide variety of collapse solutions for the collapse problem in star formation. These solutions describe the collapse of idealized molecular cloud cores and/or the collapse of kernels, the subcondensations within large cores that form stellar groups and clusters. Recent observations of star forming regions (e.g., Lee et al. 1999, 2001) indicate that the outer boundary condition for the collapse flow is not static; instead, the collapse flow must match smoothly onto a subsonic, but finite, velocity field at large radii. This paper shows that a wide variety of self-similar solutions have small but finite velocity fields at large radii, and we argue that solutions of this type provide a better description of protostellar collapse. The most important ramification of this change in the outer boundary condition is that the mass infall rates will be larger for cores with nonzero flows. We have quantified this change using the semi-analytic technique of finding similarity solutions; we have verified this result using a full numerical treatment of collapse and have derived analytic bounds/estimates to provide greater physical understanding 
of the process. All three methods are in good agreement and show how the infall rates depends on the parameters of the problem. Our specific results are listed below:

[1] In general, the equation of state that describes the thermodynamics of the collapse flow - that which determines the evolution of entropy - need not be the same as the effective equation of state that sets up the initial configuration. For the case of polytropic equations of state, this generalization means that the dynamic index $\gamma$ need not be the same as the static index $\Gamma$. This paper shows that similarity solutions can be found for the more general problem containing two equations of state. In addition, although previous work has focused on collapse flows that match onto static outer boundary conditions, this paper shows that gaseous spheres with inward flows at large radii also can be described by similarity solutions; we parameterize this latter generalization by introducing the reduced speed $v_{\infty}$ (the speed at $x \rightarrow \infty$ ). Including the overdensity parameter $\Lambda$, which has been considered previously, this paper shows that self-similar collapse solutions exist for a four parameter family of conditions, where points in this parameter space can be written in the form $\left(\Gamma, \gamma, \Lambda, v_{\infty}\right)$.

[2] The initial hydrostatic equilibrium configuration is determined by the static equation of state. In order for equilibrium configurations to exist, the index of the static equation of state must satisfy the constraint $\Gamma<4 / 3$. The power-law index $\tilde{\mu}$ of the density profile is given by

$$
\tilde{\mu}=-\frac{2}{2-\Gamma} .
$$

This result shows that the equilibrium density distribution becomes less centrally concentrated as the static equation of state becomes softer (as the static index $\Gamma$ decreases).

[3] The time dependence of the mass infall rate also depends only on the static equation of state and is given by

$$
\dot{M} \sim t^{3(1-\Gamma)} .
$$

As a result, static equations of state that are stiffer than isothermal produce mass infall rates that decrease with time. Similarly, static equations of state that are softer than isothermal lead to mass infall rates that increase with time. Since we expect the equilibrium configurations of actual cloud cores to correspond to the softer equations of state, the mass infall rate should either remain constant (as for the isothermal case) or increase with time. This result holds for any value of dynamic $\gamma$.

[4] Collapse solutions which approach a ballistic free-fall form in the inner region $(x \ll 1)$ exist for all dynamic equations of state with indices $\gamma<5 / 3$. For these solutions, the reduced fluid fields approach the asymptotic forms

$$
m \sim m_{0}, \quad v \sim-\sqrt{2 m_{0}} x^{-1 / 2}, \quad \alpha \sim(4-3 \Gamma) \sqrt{m_{0} / 2} x^{-3 / 2},
$$

in the limit $x \rightarrow 0$. The reduced mass $m_{0}$ sets the magnitude of the mass infall rate. As shown by equation (31), the infall rate is determined, except for the reduced mass, by the similarity transformation. 
[5] For collapse starting from an isothermal static equation of state (which implies $\rho \sim r^{-2}$ ), we have solved the full equations of motions (the original partial differential equations) in addition to the similarity equations. These numerical results provide important confirmations of the similarity approach: (A) The full numerical treatment verifies the solutions found semi-analytically; in particular, they predict the same mass infall rates. (B) The numerical results show that non-singular initial configurations, in particular those that have a central region of constant density, approach the similarity solutions asymptotically in time. Moreover, the convergence occurs relatively rapidly and smoothly, especially for the observationally preferred cases with $\Lambda>1$ and $v_{\infty} \neq 0$.

[6] We have derived analytic estimates for the reduced mass $m_{0}$ which sets the mass infall rate through equation (31). For isothermal starting states, we obtain analytic expressions for $m_{0}$ as a function of overdensity $\mathcal{A}$ (see equation [56]) and as a function of the initial inward speed $v_{\infty}$ (see equation [61]). These expressions predict the same infall rates calculated from both the numerical treatment (solving the partial differential equations) and our semi-analytic approach (the similarity solutions) as shown in Figures 6 and 7. For the case of non-isothermal initial conditions, we have derived an analytic expression for $m_{0}$ as a function of the index $\Gamma$ (see equation [79]) for collapse with $\gamma=\Gamma$. The resulting expression is in good agreement with the values calculated from the similarity solutions (Figure 10).

[7] For collapse solutions with $\gamma \neq \Gamma$, the infall rates are esssentially the same when the two indices are comparable (see Figures 1 and 11). For soft equations of state $\Gamma \ll 1$, however, the infall rates are greatly suppressed for relatively large values of dynamic $\gamma \sim 1$ (see Figure 12). In the logatropic limit for the static equation of state, $\Gamma \rightarrow 0$, this formulation does not admit self-similar solutions of the first kind for arbitrary dynamic $\gamma$.

[8] The observational motivation for this study is that starless cores can exhibit inward motions at about half the sound speed, $u_{i n} \approx a_{s} / 2$. These motions could arise from two conceptually different sources. If the core is overdense $\Lambda>1$, then the entire core - including the outer regions - will begin to collapse, with observable inward motions appearing on the outside before an observable infrared source appears in the center. Alternatively, the inward motions could be part of the initial conditions - they could be present at the effective zero point of time (the start of collapse), presumably left over from the core formation process. Both cases are compatible with current observations, although the overdense scenario is more constrained. The net result, for both scenarios, is that the infall

rate with nonzero initial velocities is larger (than in the hydrostatic case) by a factor of two (see also Allen et al. 2003b).

\subsection{Discussion}

The specific results outlined in $\S 6.1$ represent a significant mathematical generalization of the self-similar collapse problem, which now includes the four parameter family of solutions given by $\left(\Gamma, \gamma, \Lambda, v_{\infty}\right)$. Moreover, the analytic expressions derived for $m_{0}(\Lambda), m_{0}\left(v_{\infty}\right)$, and $m_{0}(\Gamma)$ provide 
both a convenient means of obtaining the infall rates (through equation [31]) and a path toward greater physical understanding of the infall process. This work has additional astronomical implications, however, as we discuss next.

The generalized collapse solutions presented here are motivated by astronomical observations which indicate that cores have inward motions at large radii at the start of the collapse phase (Lee et al. 1999, 2001). The first implication of these new solutions is that molecular cloud cores will collapse with larger mass infall rates due to these inward velocities. When matched to the observed boundary conditions, our collapse solutions imply that the infall rates will be a factor of two larger than previous estimates.

Even with the previous (smaller) infall rates, observed protostellar objects have smaller luminosities than those predicted by theoretical infall rates (e.g., Kenyon \& Hartmann 1995), unless a great deal of the energy is stored as orbital motions. With the higher infall rates now indicated, this luminosity problem is worse by a factor of two, which means that even more energy must be stored as rotational energy in the disk. With increased infall rates, the predicted time scales for star formation are shorter by this same factor of two. As argued from both observational (Myers \& Fuller 1992) and theoretical (Adams \& Fatuzzo 1996) considerations, stars form on a time scale $t_{s f} \approx 10^{5}$ years over a wide range of stellar masses. Although the mean time is shorter, the prediction that the distribution of time scales is much narrower than the distribution of stellar masses (the IMF) remains the same.

The theoretical picture of star formation developed in the 1980s considered molecular cloud cores to be (nearly) hydrostatic at the beginning of the collapse phase. More recent observations of the numbers of starless cores (e.g., Jijina et al. 1999) indicate that core formation must take place more rapidly and is unlikely to involve a purely quasi-static process. Some modification to the picture is thus necessary. Many recent authors (see the review of MacLow \& Klessen 2004) have been exploring the opposite limit in which the star formation process is fully dynamic and that cloud cores never really form at all (at least not as separate, physically well-defined entities). The picture of star formation emerging from this study is intermediate between these two extremes: Cores form relatively quickly (through physical processes not calculated here), but still represent the initial conditions for the following stage of protostellar collapse. With their rapid formation, the cores display non-zero inward motions at large radii as observed. When this dynamic element is incorporated into the collapse solutions (as calculated in this paper), the subsequent collapse proceeds much as in previous models but with a larger infall rate (due to the head start provided by the initial velocities). As a result, the scenario retains the successes of the standard picture (Shu et al. 1987), such as the spectral energy distributions of young stellar objects (Adams et al. 1987) and their corresponding observed emission maps (Walker et al. 1990). At the same time, the picture can be made consistent with faster formation times for cloud cores (Jijina et al. 1999) and the observed inward motions at large radii (Lee et al. 1999).

This modification to the initial conditions - inward velocities due to either overdense starting 
states or nonzero $v_{\infty}$ as an initial condition - alleviates one theoretical difficulty associated with protostellar collapse, but raises another. On the positive side, the inward velocities ensure that non-singular cores collapse smoothly, and more rapidly approach the self-similar solutions. On the negative side, given their dynamic formation, the cores no longer display a clean separation between their formation stage and their collapse stage.

In the (old) hydrostatic picture, molecular cloud cores live on the edge between collapse and expansion. For example, in the isothermal case (Shu 1977) where $\rho_{0}=\mathcal{A} a_{s}^{2} / 4 \pi G r^{2}$ and $\mathcal{A}=2$ corresponds to equilibrium, cores with $\mathcal{A} \geq 2$ will collapse but cores with $\mathcal{A}<2$ will actually expand! If cores were formed in states indistinguishable from the hydrostatic equilibrium configuration with $\mathcal{A}=2$, then they would always be in danger of expanding. In retrospect, it seems unlikely that the fate of a cloud core - to collapse or to expand - should depend with hair-trigger sensitivity on whether $\mathcal{A}=2+\epsilon$ or $\mathcal{A}=2-\epsilon$ (where $\epsilon \ll 1$ ). In contrast, cores with initial inward velocities (as now observed) will collapse under a robust set of circumstances.

The initial inward velocities also affect the issue of cores with constant density centers (as observed by Ward-Thompson et al. 1999, Bacmann et al. 2000, Tafalla et al. 2004). For flat-topped cores that start close to hydrostatic equilibrium, realistic collapse flows approach the self-similar form when the ratio $r_{\text {out }} / r_{C} \gtrsim 20$ (Foster \& Chevalier; Whitworth et al. 1996). Roughly speaking, the central (constant density) region collapses first, on a time scale given by $\Delta \tau \approx\left(3 \pi / 32 G \rho_{0}\right)^{1 / 2}$ (e.g., Spitzer 1978). For later times $t>\Delta \tau$, the flow approaches the self-similar form as long as more of the cloud exists to subsequently collapse (this condition holds for $r_{\text {out }} / r_{C} \gtrsim 20$ ). For cores with initial velocities, however, the time required for the central (constant density) region to collapse is reduced (as calculated in equation [52]) so that the requirement on the ratio $r_{\text {out }} / r_{C}$ is less constraining. In order words, because observed cores have initial inward velocities, the selfsimilar solutions provide a better description of the collapse flow (than if the cores were exactly hydrostatic).

An explicit demonstration of this trend is shown in Figure 13, which plots the mass infall rates for a set of flat-topped cores as a function of time. This set of numerical collapse calculations begins with isothermal cores with sound speed $a_{S}=0.2 \mathrm{~km} / \mathrm{s}$ and an extended central region of nearly constant density spanning $r_{C} \approx 10^{16} \mathrm{~cm}$. The initial states are slightly overdense, with $\mathcal{A}=2.2$. The solid curve shows the resulting mass infall rate (expressed in $M_{\odot} \mathrm{yr}^{-1}$ ) for a starting state with no initial velocity. As found previously, the infall rate is essentially zero to begin with, and then exhibits a sharp spike at the time when the constant density region reaches the origin; the infall rate then decreases with time and eventually approaches the asymptotic value $\dot{M}=m_{0} a_{S}^{3} / G$ predicted by the similarity solution. The dotted (dashed) curve shows the corresponding mass infall rates for starting inward speeds of $u_{i n}=0.5 a_{S}\left(u_{i n}=a_{S}\right)$. When $u_{i n} \neq 0$, the mass infall rates reach their peak values sooner and they more rapidly approach their asymptotic values (which are within about 10 percent of those predicted by the self-similar calculations). In addition, for cores with $u_{\text {in }} \neq 0$, the maximum infall rates are smaller and the asymptotic values are larger, so the time variability is less severe. More specifically, the ratio of the peak infall rate to its asymptotic 
value is $\sim 8.4$ for $u_{i n}=0$ and only $2-3$ for $u_{i n} \neq 0$. It has been suggested (e.g., Ward-Thompson et al. 1999) that the spike in the infall rate corresponds to the Class O phase of protostellar evolution. Although this work suggests that initial inward velocities smooth out the spike to some degree, the infall rates are nonetheless larger early on and can correspond to the Class 0 phase.

The new difficulty raised by the presence of initial inward speeds has two components. First, the physical processes that explains core formation must be able to account for the observed speeds. Second, the core formation epoch must be more dynamic than considered previously, and the distinction between the core formation stage of evolution and the core collapse stage is not as clean. While this paper clarifies the collapse phase of star formation, the (earlier) core formation phase requires further theoretical work.

Finally, one must keep in mind that this work generalizes spherical collapse solutions in the absence of magnetic fields and angular momentum. Nonrotating solutions are expected to apply

at larger radii where the flow is nearly spherical. The effects of rotation become important in the inner part of the flow, where our solutions can be matched onto inner solutions as outlined in $§ 3.5$. The inclusion of magnetic fields into the collapse problem has been studied in a variety of related contexts (see Galli \& Shu 1993ab; Li \& Shu 1996, 1997; Allen et al. 2003ab; Shu et al. 2004), including one collapse calculation with nonzero initial velocities (see Figure 7 of Allen et al. 2003b). With the inclusion of rotation (at rate $\Omega$ ) and magnetic fields $\mathbf{B}$, one is left with a six parameter family of initial conditions $\left(\Gamma, \gamma, \Lambda, v_{\infty}, \Omega, \mathbf{B}\right)$, where the velocity field $\mathbf{v}_{\infty}(\mathbf{r})$, the rotation profile $\Omega(\mathbf{r})$, and the magnetic field strength $\mathbf{B}(\mathbf{r})$ can all be functions of the spatial coordinates. With this paper (which explores collapse with $v_{\infty} \neq 0$ and $\gamma \neq \Gamma$ ), the effects of these parameters on collapse have all been considered individually. The challenge for the future is thus to identify the portion of this parameter space that applies to protostellar collapse and to properly incorporate all of the relevant effects (simultaneously) into the collapse solutions.

\section{Acknowledgments}

We would like to thank Susana Lizano and Frank Shu for early discussions concerning selfsimilar collapse and the effects of varying equations of state. We also thank Daniele Galli and Joan Najita for intermediate term discussions. Finally, we thank Gus Evrard and Greg Laughlin for more recent input, especially regarding numerical issues, and we thank the referee for a prompt and insightful report. This work was supported by the University of Michigan through the Michigan Center for Theoretical Physics, by NASA through the Astrophysics Theory Program, and by Xavier University through the Hauck Foundation. 


\section{APPENDIX: EXISTENCE OF FREE-FALL SOLUTIONS}

In this appendix, we show that ballistic free-fall solutions exist in the inner regime of the collapse flow for any dynamic equation of state with index $\gamma<5 / 3$.

We begin by taking the limiting form of the equations of motion for the case

$$
x \ll|v|, \quad x \ll 1,
$$

which defines the inner regime of the collapse flow. In this limit, the general expressions for the mass $m(x)$ and the pressure $p(x)$ reduce to

$$
m(x)=\frac{v x^{2} \alpha}{3 a+2}
$$

and

$$
p(x)=\mathcal{C}_{0} \alpha^{\gamma}\left(\alpha v x^{2} / a\right)^{q}=\mathcal{C}_{1} \alpha^{\gamma} m^{q}
$$

Next, we note that the final equation of motion takes the form

$$
\frac{1}{\alpha} \frac{d \alpha}{d x}+\frac{1}{v} \frac{d v}{d x}=-\frac{2}{x}
$$

which can be immediately integrated to obtain

$$
\alpha v x^{2}=\text { constant }
$$

Comparing this result with the limiting form for the mass $m(x)$ shows that the enclosed mass always approaches a constant value in the inner limiting regime, i.e.,

$$
m(x) \rightarrow m_{0}=\text { constant }
$$

This result is quite general; it was obtained by assuming only that the coordinate $x$ is small (which defines what we mean by the inner regime of the flow) and by assuming that $x \ll|v|$. This latter assumption implies that we are considering only infall solutions in this argument. As a general rule, outflow solutions to the equations of motion will also often exist. The outflow solutions generally have $v \rightarrow 0$ and $x \rightarrow 0$ and hence the results of this current argument are not applicable.

Given that the reduced mass approaches a constant value in the inner regime, the reduced pressure can be simplified also, i.e.,

$$
p(x) \rightarrow \widetilde{\mathcal{C}} \alpha^{\gamma},
$$

where we have defined a new constant $\widetilde{\mathcal{C}}$. This result makes sense: it means that the pressure is given by a pure polytropic form in the inner limit, where the polytropic index is determined by the dynamic equation of state. In this limit, the gas has lost all memory of the original "static" equation of state. 
The equation of motion (the force equation) reduces to the form

$$
v \frac{d v}{d x}+\gamma \widetilde{\mathcal{C}} \alpha^{\gamma-2} \frac{d \alpha}{d x}+\frac{m_{0}}{x^{2}}=0 .
$$

Our objective is to show that a self-consistent free-fall solution exists for certain values of the index $\gamma$. If a free-fall solution exists, then the velocity must approach the form

$$
v=-\left(2 m_{0}\right)^{1 / 2} x^{-1 / 2}
$$

where the minus sign indicates that the material is falling inward. Using this form for the velocity in the equation of motion (90), we see that the first velocity term exactly balances the third gravity term. This solution will exist provided that the second pressure term vanishes. Since $m \rightarrow m_{0} \sim v x^{2} \alpha$ in this limit, $\alpha \sim x^{-3 / 2}$. The ratio $\mathcal{R}$ of the pressure term to the gravity term thus becomes

$$
\mathcal{R} \sim \text { constant } \times x^{(5-3 \gamma) / 2} .
$$

This ratio vanishes in the limit of small $x$ provided that

$$
\gamma<5 / 3
$$

Thus, self-consistent free-fall solutions exist in the inner limit for all dynamic equations of state with indices $\gamma<5 / 3$. 


\section{REFERENCES}

Adams, F. C. 1990, ApJ, 363, 578

Adams, F. C., \& Fatuzzo, M. 1996, ApJ, 464, 256

Adams, F. C., Lada, C. J., \& Shu, F. H. 1987, ApJ, 321, 788

Allen, A., Li, Z.-Yi., \& Shu, F. H. 2003a, ApJ, 599, 363

Allen, A., Shu, F. H., \& Li, Z.-Yi. 2003b, ApJ, 599, 351

Bacmann, A., André, P., Puget, J.-L., Abergel, A., Bontemps, S., \& Ward-Thompson, D. 2000, A\&A, 361, 555

Barenblatt, G. I. 2003, Scaling (Cambridge: Cambridge Univ. Press)

Benson, P. J., \& Myers, P. C. 1987, ApJS, 71, 89

Boss, A. P., \& Myhill, E. A. 1992, ApJSS, 83, 311

Cassen, P., \& Moosman, A. 1981, Icarus, 48, 353

Chandrasekhar, S. 1939, Stellar Structure (New York: Dover)

Cheng, A. F. 1977, ApJ, 213, 537

Cheng, A. F. 1978, ApJ, 221, 320

Chevalier, R. A. 1983, ApJ, 268, 753

Ciolek, G. E., \& Basu, S. 2001, ApJ, 547, 272

Curry, C., \& McKee, C. F. 2000, ApJ, 528, 734

Curry, C., \& Stahler, S. W. 2001, ApJ, 555, 160

Fatuzzo, M., \& Adams, F. C. 2002, ApJ, 570, 210

Foster, P. N., \& Chevalier, R. A. 1993, ApJ, 416, 303

Galli, D., \& Shu, F. H. 1993a, ApJ, 417, 220

Galli, D., \& Shu, F. H. 1993b, ApJ, 417, 243

Hunter, C. 1977, ApJ, 218, 834

Jijina, J., \& Adams, F. C. 1996, ApJ, 462, 874

Jijina, J., Myers, P. C., \& Adams, F. C. 1999, ApJ Suppl., 125, 161

Kenyon, S. J., \& Hartmann, L. W. 1995, ApJS, 101, 117

Larson, R. B. 1969a, MNRAS, 145, 271 
Larson, R. B. 1969b, MNRAS, 145, 297

Larson, R. B. 1985, MNRAS, 214, 379

Lee, C. W., Myers, P. C., \& Tafalla, M. 1999, ApJ, 526, 788

Lee, C. W., Myers, P. C., \& Tafalla, M. 2001, ApJ Suppl., 136, 703

Li, Y., Klessen, R. S.,\& MacLow, M.-M. 2003, ApJ, 592, 975

Li, Z.-Y. 1999, ApJ, 526, 806

Li, Z.-Y., \& Shu, F. H. 1996, ApJ, 472, 211L

Li, Z.-Y., \& Shu, F. H. 1997, ApJ, 475, 237

Lizano, S., \& Shu, F. H. 1989, ApJ, 342, 834

Mac Low, M.-M., \& Klessen, R. S. 2004, Rev. Mod. Phys., 76, 125

McLaughlin, D. E., \& Pudritz, R. E. 1997, ApJ, 476, 750

Motte, F., \& André, P. 2001, A\&A, 365, 440

Mouschovias, T. Ch. 1976, ApJ, 207, 141

Myers, P. C. 1998, ApJ, 496, L109

Myers, P. C. 2004, submitted to ApJ

Myers, P. C., \& Benson, P. J. 1983, ApJ, 266, 309

Myers, P. C., \& Fuller, G. A. 1992, ApJ, 396, 631

Myers, P. C., \& Lazarian, A. 1998, ApJ, 507, L157

Nakano, T. 1984, Fund. Cosmic Phys., 9, 139

Penston, M. V. 1969a, MNRAS, 144, 425

Penston, M. V. 1969b, MNRAS, 145, 457

Shu, F. H. 1977, ApJ, 214, 488

Shu, F. H. 1983, ApJ, 273, 202

Shu, F. H., Adams, F. C., \& Lizano, S. 1987, A R A \& A, 25, 23

Shu, F. H., Li, Z.-Y., \& Allen, A. 2004, ApJ, 601, 930

Spaans, M., \& Silk, J. 2000, ApJ, 538, 115

Spitzer, L. 1978, Physical Processes in the Interstellar Medium (New York: Wiley)

Stahler, S. W., Shu, F. H., \& Taam R. E. 1980, ApJ, 241, 637 
Tafalla, M., Myers, P. C., Caselli, P., \& Walmsley, C. M. 2004, A\&A, 416, 191

Terebey, S., Shu, F. H., \& Cassen, P. 1984, ApJ, 286, 529

Ulrich, R. K. 1976, ApJ, 210, 377

Vazquez-Semadeni, E., Canto, J., \& Lizano S. 1998, ApJ, 492, 596

von Weizsäcker, C. F. 1954, Z. Naturforschung, 9A, 269

Walker, C. J., Adams, F. C., \& Lada, C. J. 1990, ApJ, 349, 515

Ward-Thompson, D., Motte, F., \& André, P. 1999, MNRAS, 305, 143

Whitworth, A., \& Summers, D. 1985, MNRAS, 214, 1

Whitworth, A. P., Bhattal, A. S., Francis, N., \& Watkins, S. J. 1996, MNRAS, 283, 1061

Wolfire, M. G., \& Cassinelli, J. 1986, ApJ, 310, 207

Wolfire, M. G., \& Cassinelli, J. 1987, ApJ, 319, 850

Zeldovich, Ya. B. 1956, Sov. Phys. Acoustics, 2, 25

Zweibel, E. G. 2002, ApJ, 567, 962 


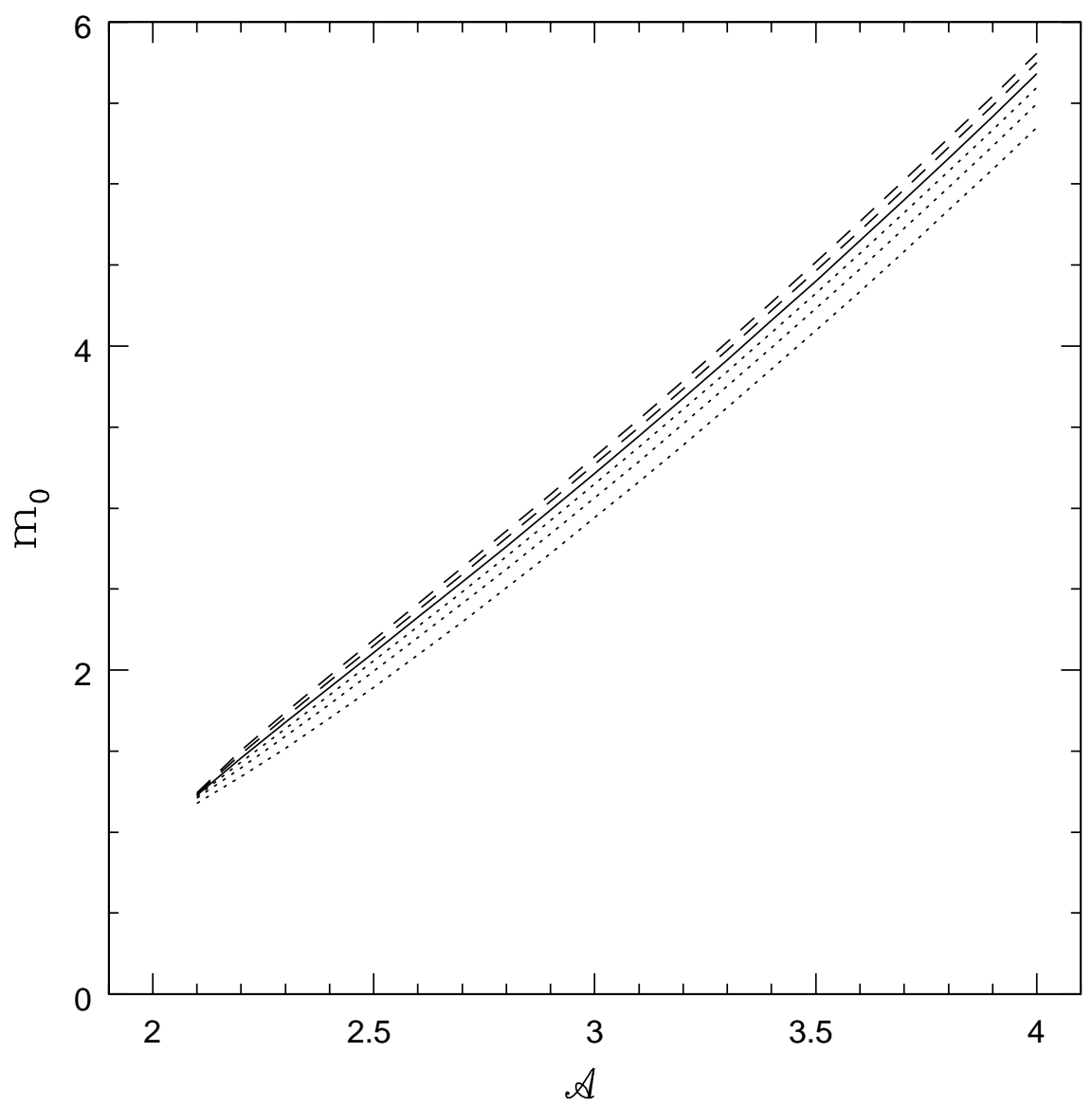

Fig. 1.- For initial states with isothermal static $\Gamma=1$, the mass infall rate $\dot{M}=m_{0} a_{s}^{3} / G$. This plot shows the variation of $m_{0}$ with the overdensity of the initial configuration for various values of the dynamic $\gamma$. Hydrostatic equilibrium corresponds to $\mathcal{A}=2$. The upper dashed curves show results for $\gamma=0.6$ and $0.8(\gamma<1)$; the lower dotted curves show results for $\gamma=1.2,1.4$, and 1.6 $(\gamma>1)$; the solid curve corresponds to $\gamma=1$ (see Shu 1977). 


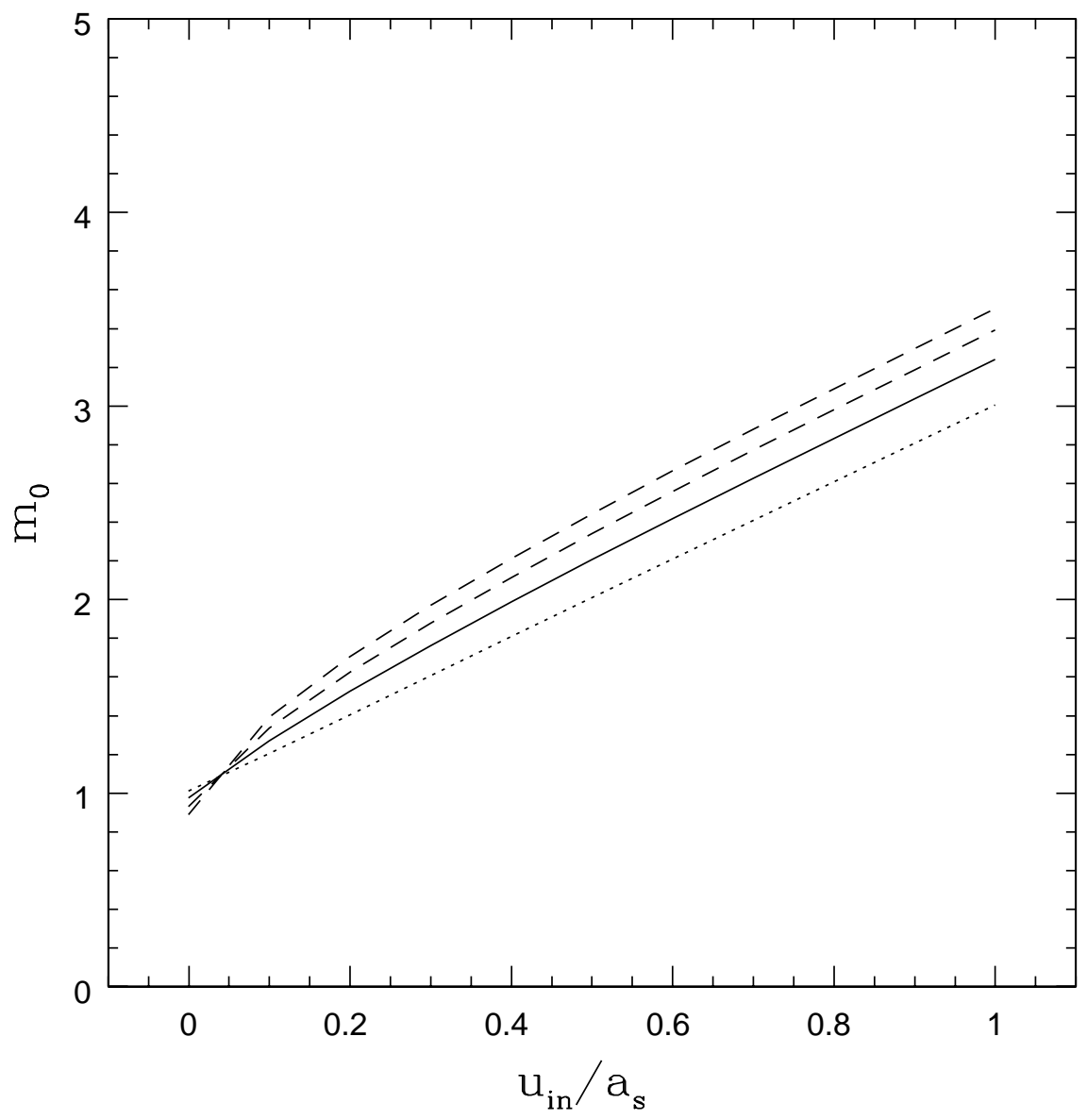

Fig. 2.- For initial states with isothermal static $\Gamma=1$, the mass infall rate $\dot{M}=m_{0} a_{s}^{3} / G$. This plot shows the variation of $m_{0}$ with the initial inward velocity $v_{\infty}=u_{i n} / a_{s}$ for various values of the dynamic $\gamma$. Hydrostatic equilibrium corresponds to $u_{i n}=0$. The upper dashed curves show results for $\gamma=0.6$ and $0.8(\gamma<1)$; the lower dotted curve shows results for $\gamma=1.2$; the solid curve corresponds to $\gamma=1$. 

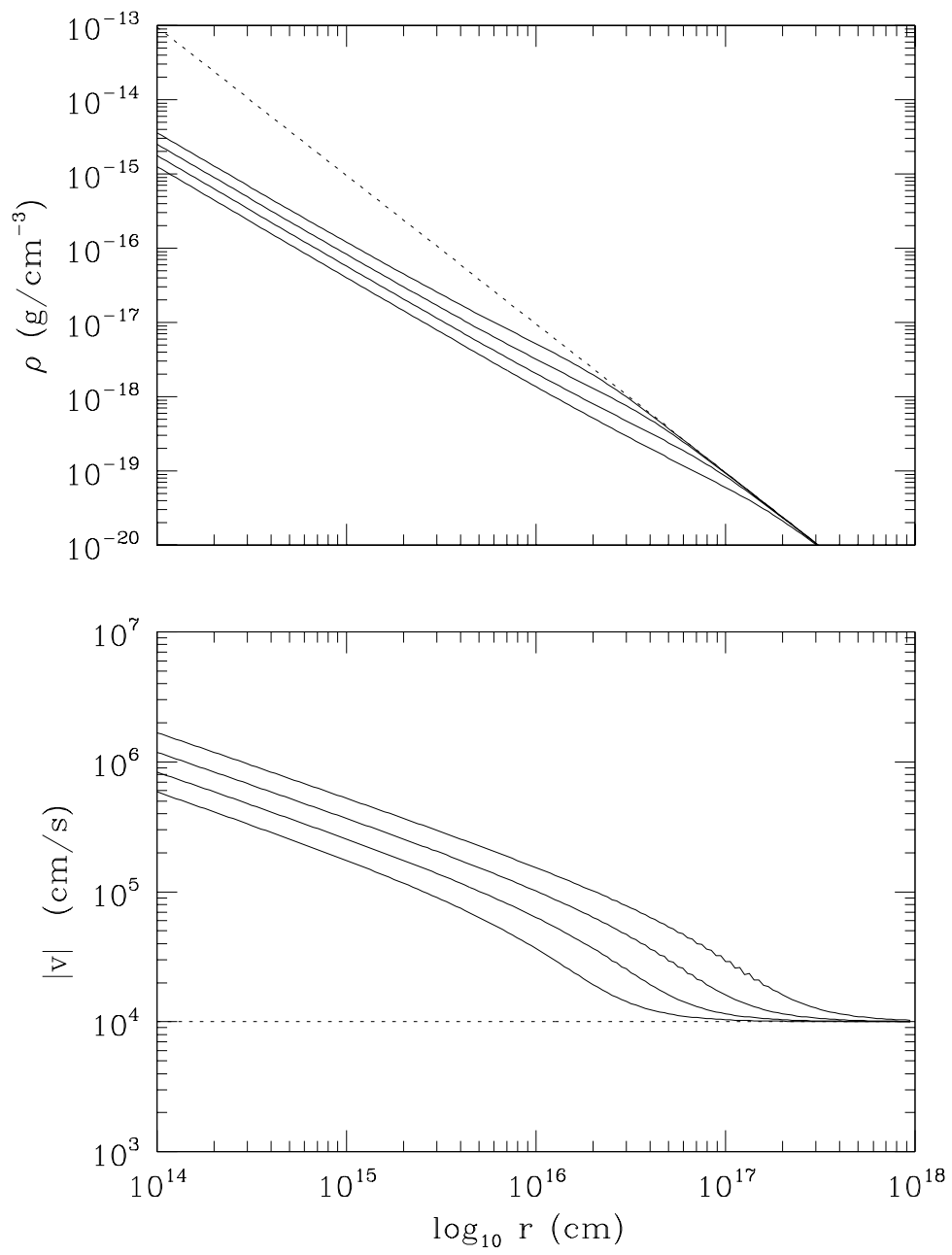

Fig. 3.- This figure shows the numerically determined collapse solution for a gaseous sphere with the density appropriate for hydrostatic equilibrium $(\mathcal{A}=2)$ and an initial inward velocity of magnitude $u_{\text {in }}=0.5 a_{s}$. The top panel shows the resulting density profile at four times (scaled to typical parameters for a molecular cloud core). The bottom panel shows the corresponding inward velocity profile at the same four epochs. Notice that $v \rightarrow$ constant in the limit $r \rightarrow \infty$ and the profiles have the same (self-similar) form at smaller radii. 

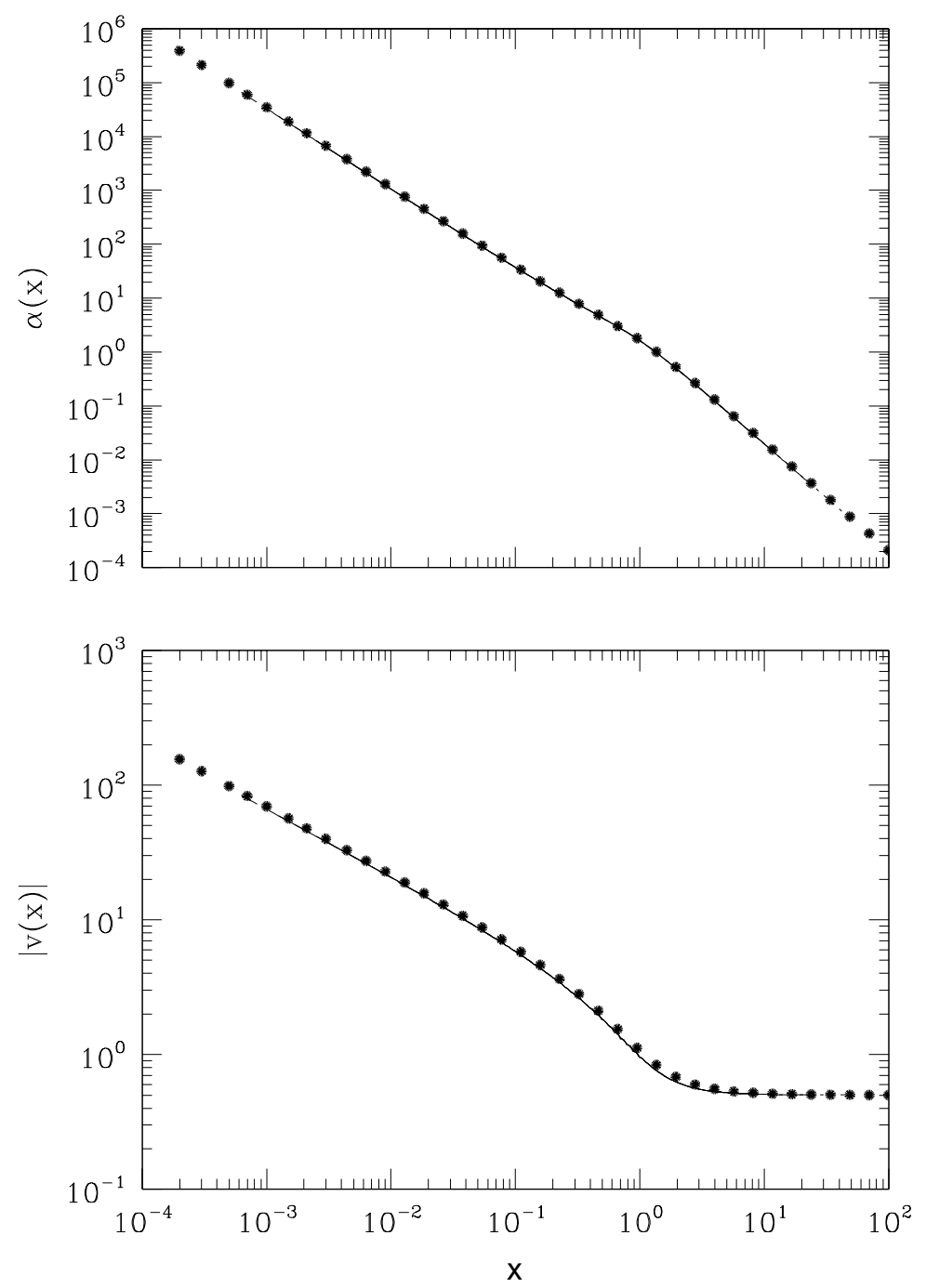

Fig. 4.- This figure illustrates how the numerically determined collapse solution for a gaseous sphere approaches a self-similar form. The numerically determined solutions for both the density (top panel) and velocity (bottom panel) are rescaled according to the (expected) similarity transformation and then plotted on top of each other. The result is one smooth function (for each panel) and hence the numerically determined solutions at any given time are a stretched version of the solution at an earlier time, in keeping with the expectation of self-similarity. The corresponding self-similar solution, shown by the filled circles, is in good agreement. 


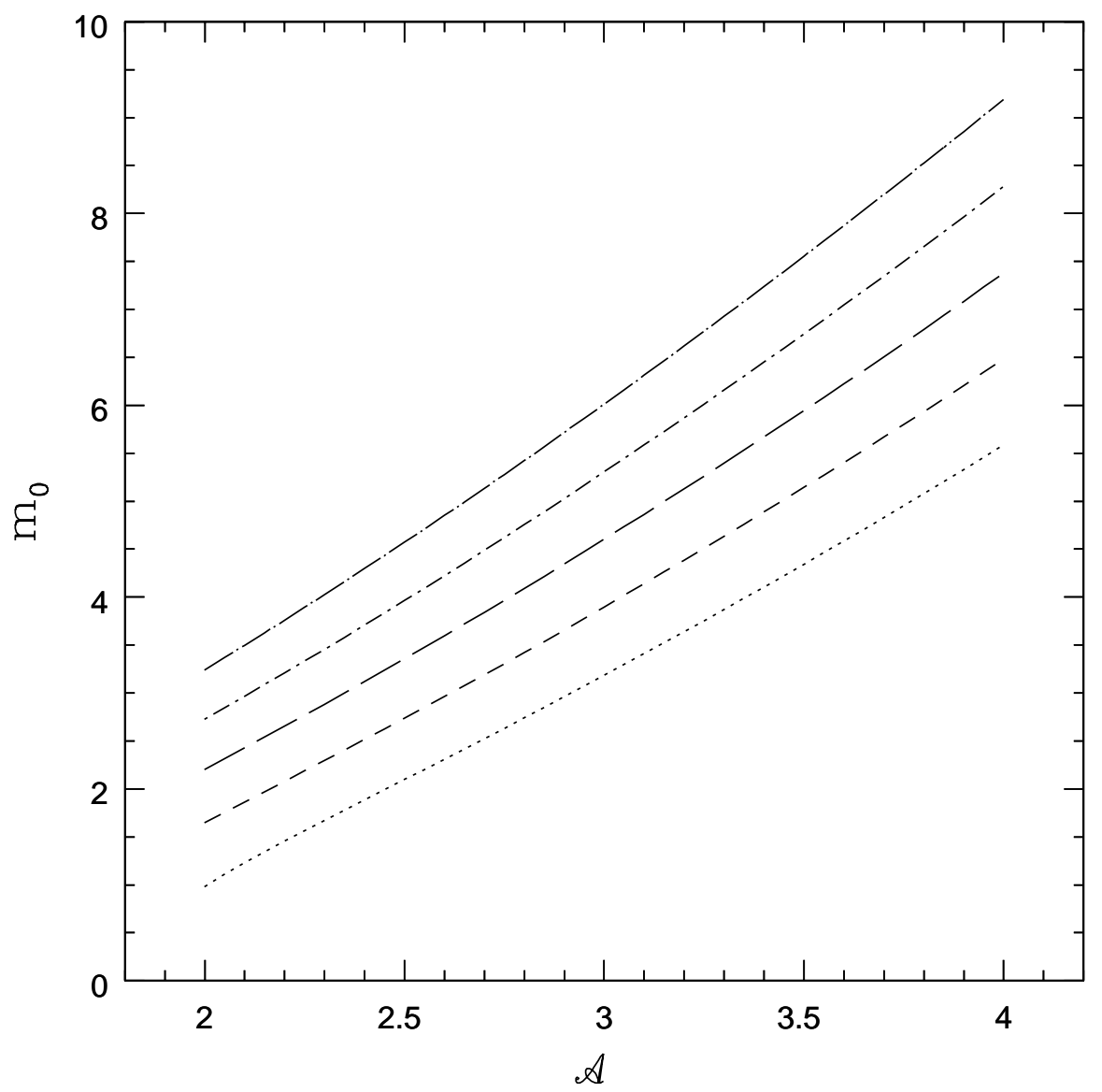

Fig. 5.- Numerically determined infall rates shown as a function of the overdensity parameter $\mathcal{A}$ and the initial (inward) velocity. All collapse models use isothermal conditions so that $\gamma=1=\Gamma$. Each curve shows the numerically calculated values of $m_{0}$ as a function of $\mathcal{A}$ for a given starting speed $u_{i n}$. The lower curve shows the result for $u_{i n}=0$. The next four curves, in ascending order, show the results for $u_{i n} / a_{s}=0.25,0.5,0.75$, and 1.0, where $a_{s}$ is the isothermal sound speed. 


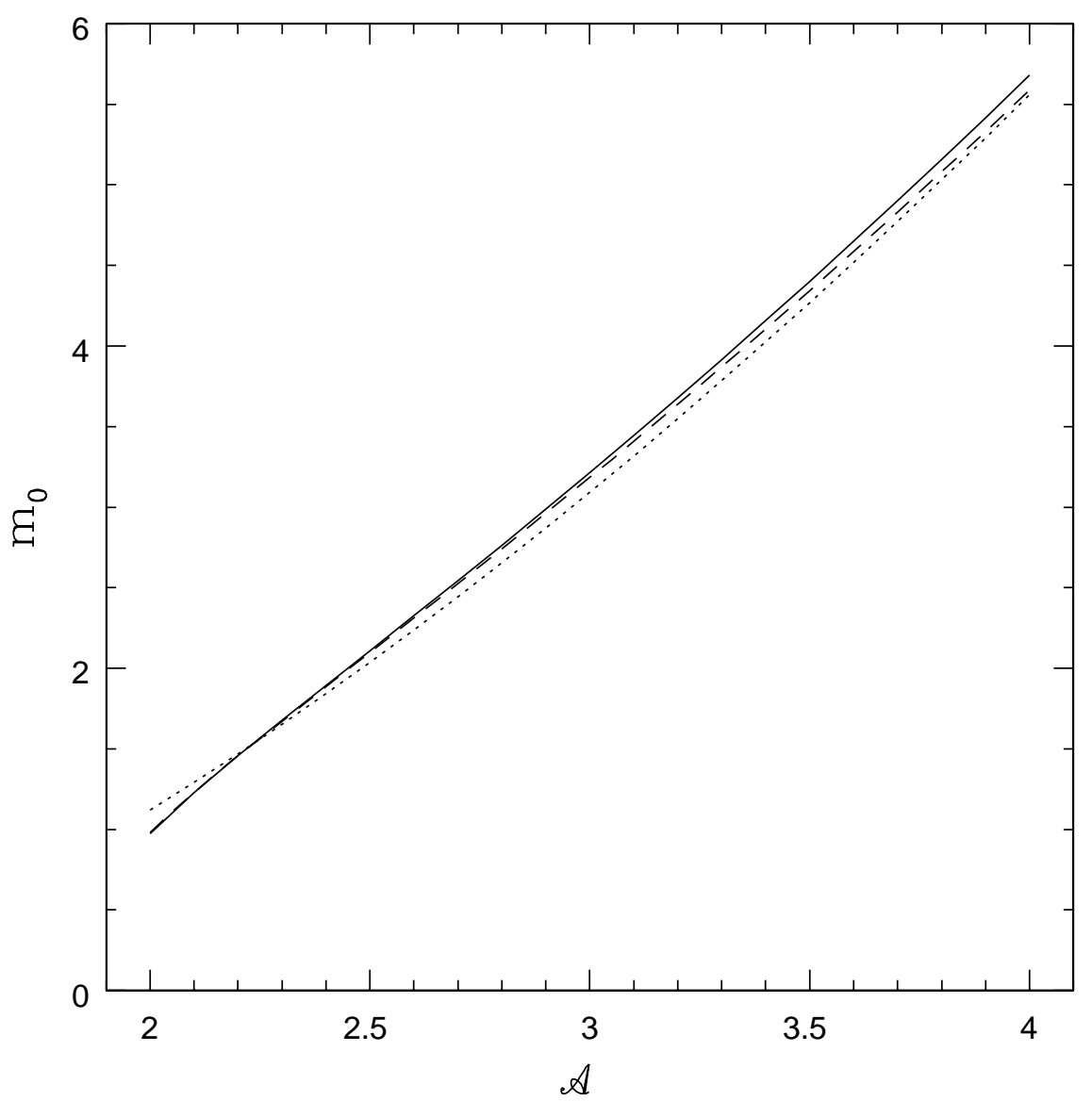

Fig. 6.- Comparison of self-similar, analytic, and numerical determinations of $m_{0}$, the dimensionless mass that specifies the infall rate for isothermal initial conditions. The analytic estimate for $m_{0}$ is shown by the dotted curve; the values calculated from the self-similar equations of motion are shown by the solid curve; the results from a numerical treatment (solving the partial differential equations) are shown as the dashed curve. All models have initial states with isothermal static $\Gamma$ $=1$ and dynamic $\gamma=1$. Keep in mind that for all of these configurations, the mass infall rate is given by $\dot{M}=m_{0} a_{s}^{3} / G$ so that $m_{0}$ specifies the infall rate. 


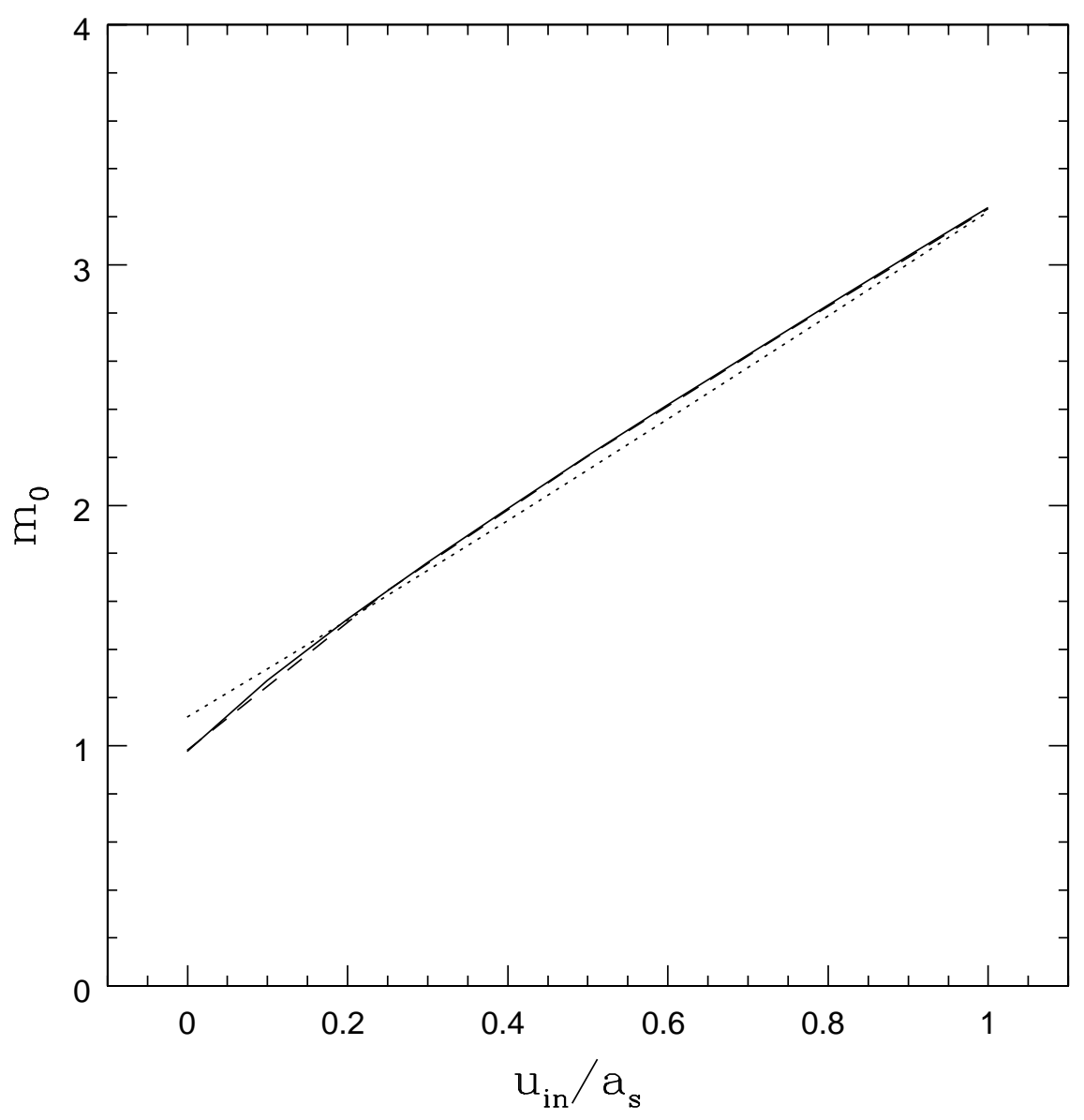

Fig. 7.- Comparison of self-similar, analytic, and numerical determinations of $m_{0}$ for varying initial inward velocities $u_{i n}$ and for isothermal initial conditions. The $m_{0}$ values calculated from the self-similar equations of motion are shown by the solid curve; the results from a numerical treatment (solving the partial differential equations) are shown as the dashed curve. The analytic estimate for $m_{0}$ is shown by the dotted curve; to obtain this form, we assume a fitting parameter that takes into account a slight (20 percent) acceleration of the flow before the expansion wave passes. All models use initial states with isothermal static $\Gamma=1$ and dynamic $\gamma=1$. For all cases, the mass infall rate is given by $\dot{M}=m_{0} a_{s}^{3} / G$ so that $m_{0}$ specifies the infall rate. 


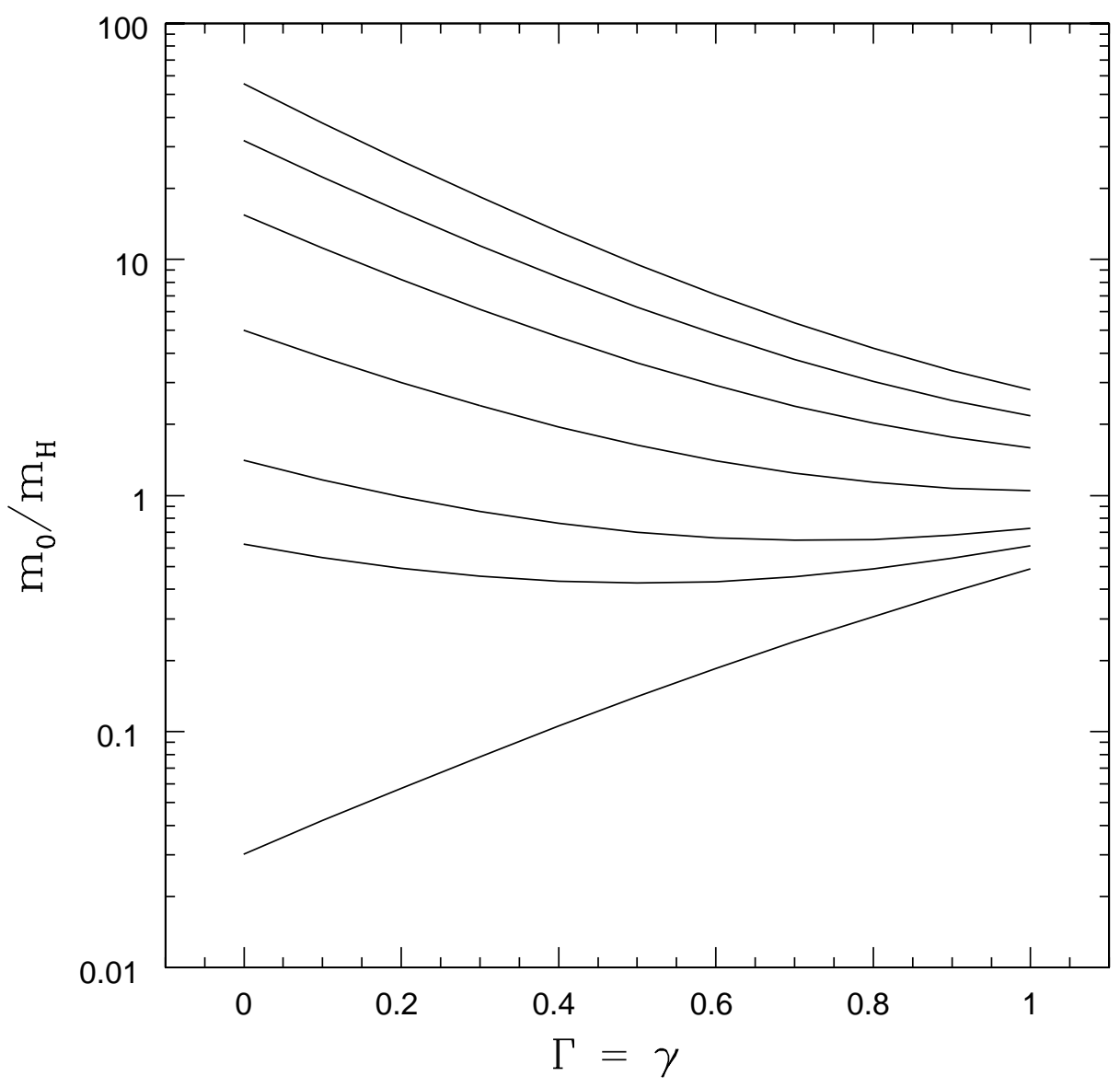

Fig. 8.- Reduced masses $m_{0}$ as a function of equation of state index $\Gamma=\gamma$ for varying values of the overdensity. The results are expressed as the ratio $m_{0} / m_{H}$, where $m_{H}$ is the reduced mass enclosed within the expansion wave for the case of hydrostatic equilibrium (the reduced masses $m_{0}$ for each overdensity is thus compared to the same $m_{H}$ ). The lowest curve shows the case of hydrostatic equilbrium with $\Lambda=1$. The subsequent curves (proceeding upward) show the results for $\Lambda=1.05,1.10,1.25,1.50,1.75$, and 2.0. Notice that the infall rate, which is proportional to $m_{0}$, varies much more rapidly with the overdensity $\Lambda$ for smaller values of index $\Gamma$. 


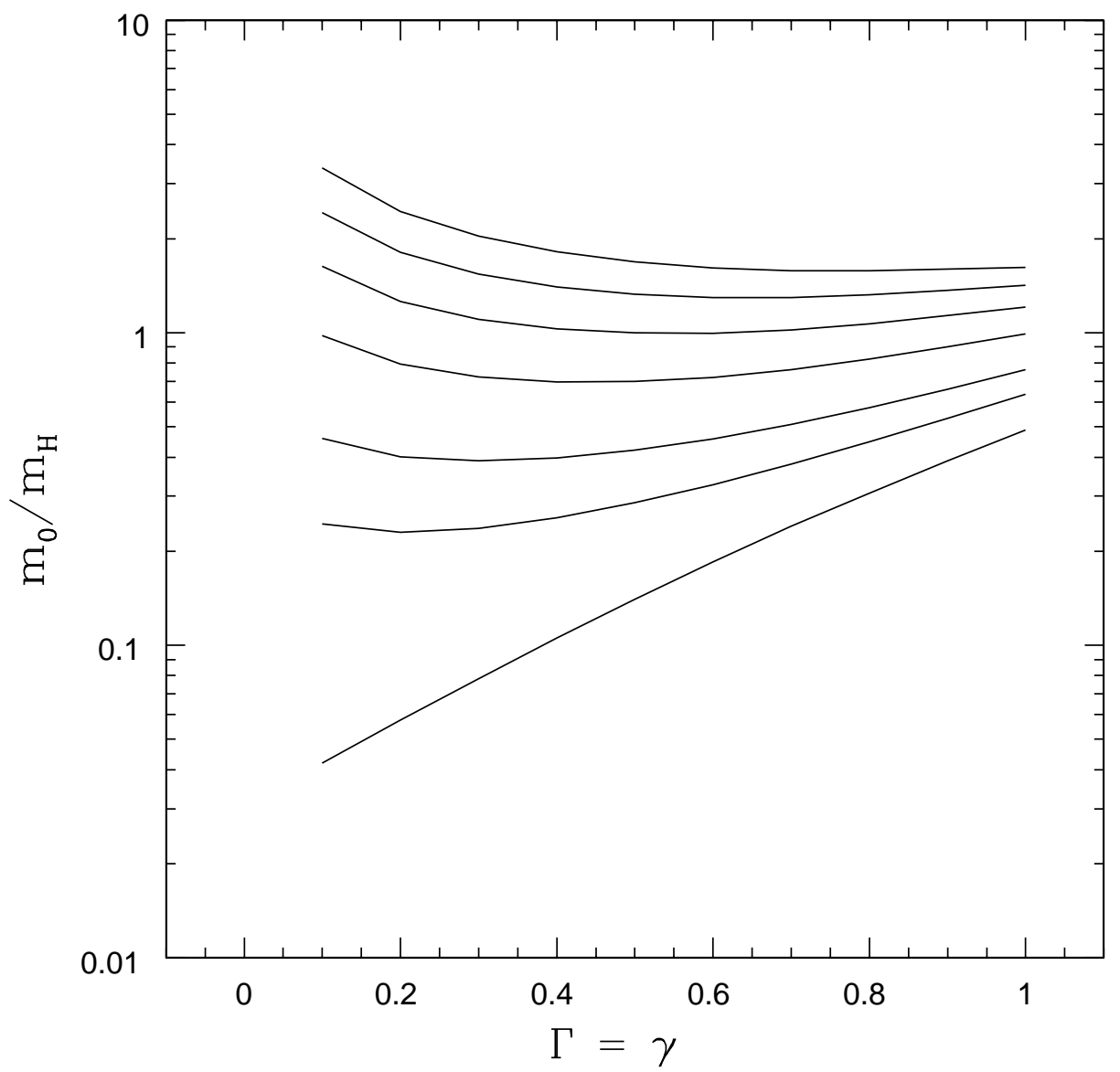

Fig. 9.- Reduced masses $m_{0}$ as a function of equation of state index $\Gamma=\gamma$ for varying values of the inward speed $v_{\infty}$ (where $v(x) \rightarrow v_{\infty}$ at large $x$ ). The results are expressed as the ratio $m_{0} / m_{H}$, where $m_{H}$ is the reduced mass enclosed within the expansion wave for the case of hydrostatic equilibrium (the reduced masses $m_{0}$ for each case is compared to the same $m_{H}$ ). The lowest curve shows the case of hydrostatic equilbrium with $v_{\infty}=0$. The subsequent curves (proceeding upward) show the results for $v_{\infty}=0.1,0.2,0.4,0.6,0.8$, and 1.0 . 


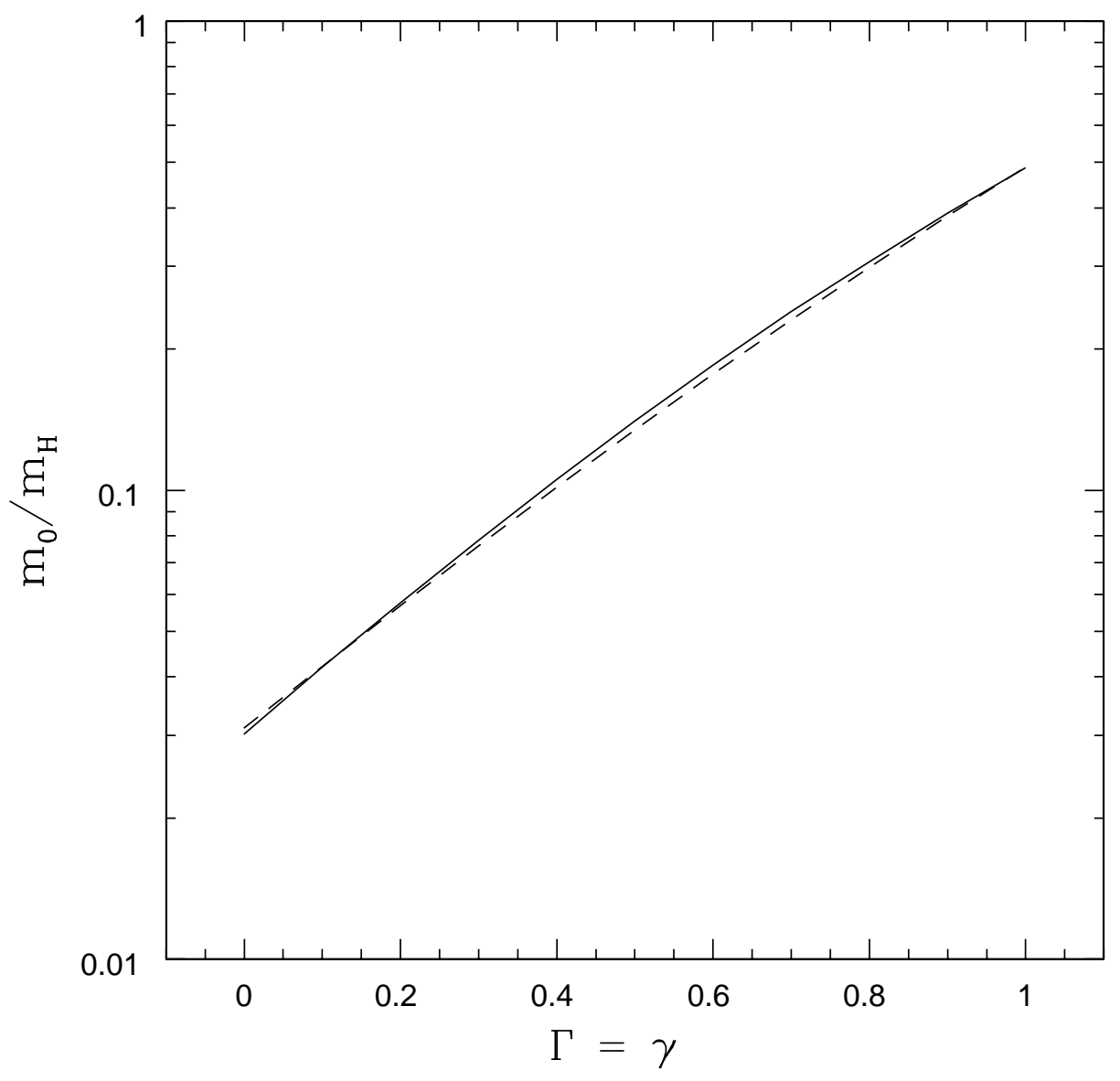

Fig. 10.- Comparison of the analytic estimates for $m_{0}$ (dashed curve) with the values calculated from the self-similar equations of motion (solid curve) for initial states with varying values of the polytropic index. In this case, the dynamic $\gamma$ is set equal to the static $\Gamma$. The mass infall rate $\dot{M}$ is proportional to $m_{0}$ (although the scalings depend on $\gamma=\Gamma$ ). The dashed curve shows the analytic result of equation $(79)$ with $g(\Gamma)=(4 / 3)(2-\Gamma)^{9 / 10}$; this form takes into account the result, found numerically, that polytropes with softer equations of state approach free-fall conditions more slowly as $\Gamma$ decreases. 


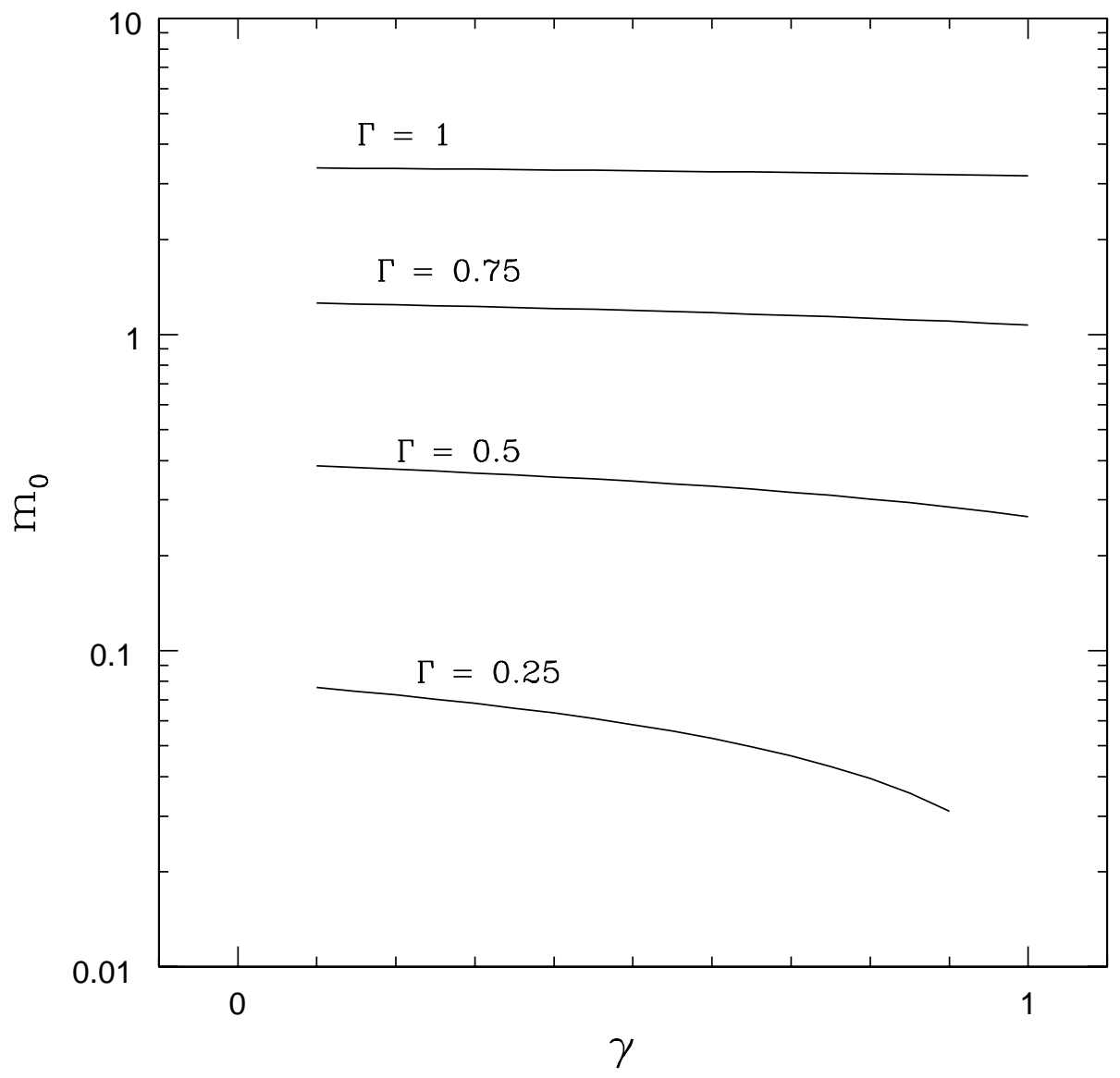

Fig. 11. - The effect of dynamic $\gamma$ on infall rates. The various curves show the value of the reduced mass $m_{0}$ (which sets the infall rate) as a function of dynamic $\gamma$ for fixed values of the static equation of state, i.e., fixed values of static $\Gamma$ (as labeled). The variation in dynamic $\gamma$ has relatively little effect unless $\gamma \gg \Gamma$; in this latter regime, the reduced masses (and infall rates) become much smaller than in the case of $\gamma=\Gamma$. 


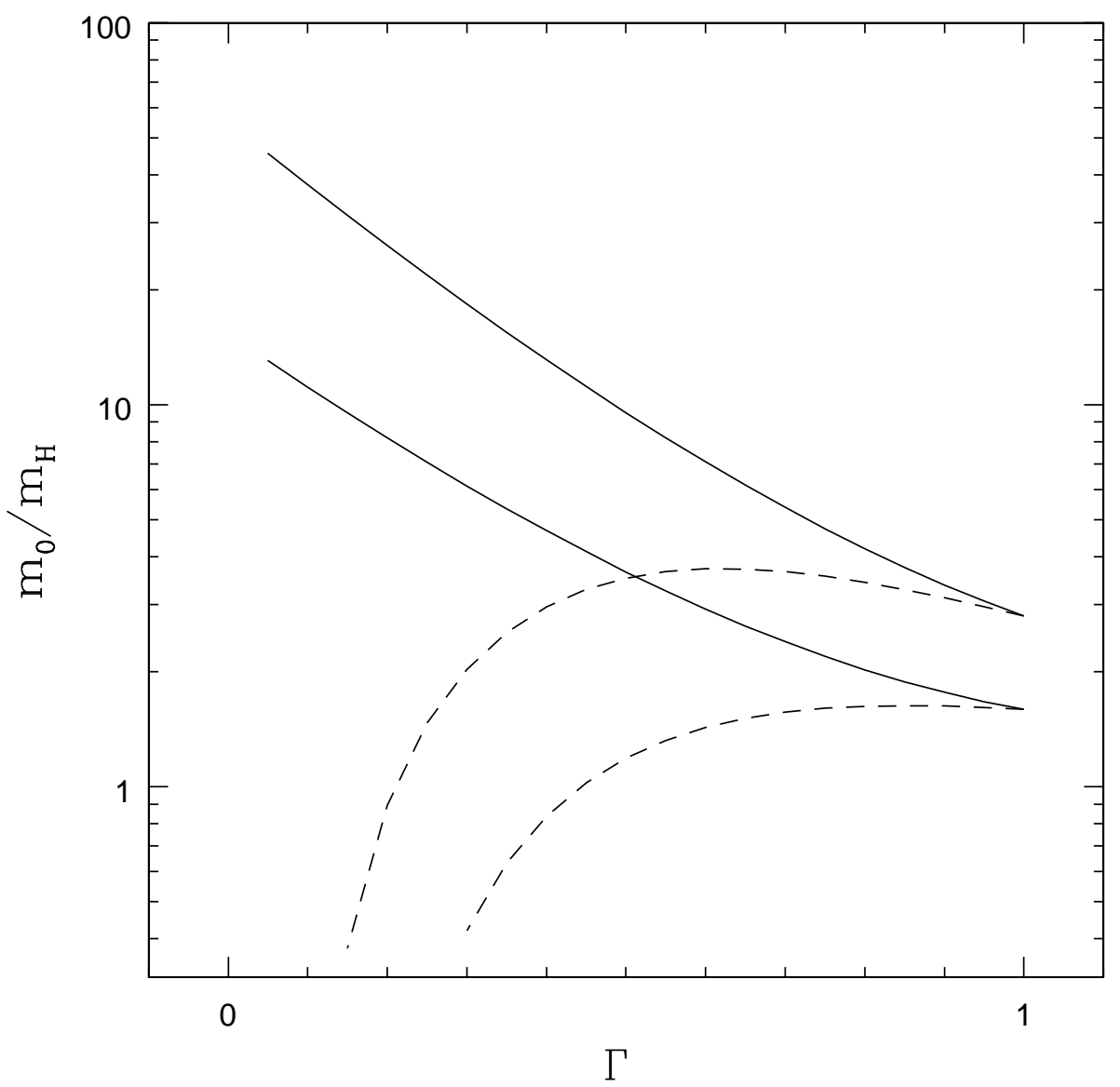

Fig. 12. - Reduces mass ratio for varying static indices $\Gamma$ and fixed (isothermal) dynamic $\gamma=1$. These curves show the numerically determined values of $m_{0} / m_{H}$, which set the mass infall rates, as a function of static index $\Gamma$ (the denominator uses the hydrostatic value of $m_{H}$ with $\Lambda=1$ ). The solid curves represent models in which the dynamic index is the same, i.e., $\gamma=\Gamma$. The dashed curves show cases where dynamic $\gamma=1$. In other words, the polytropic spheres are allowed to have varying initial density profiles (as set by varying $\Gamma$ ), but the entropy evolution of the gas takes place under isothermal conditions $\gamma=1$. The upper curves correspond to initial states with overdensity $\Lambda=2$; the lower curves use overdensity $\Lambda=1.5$. 


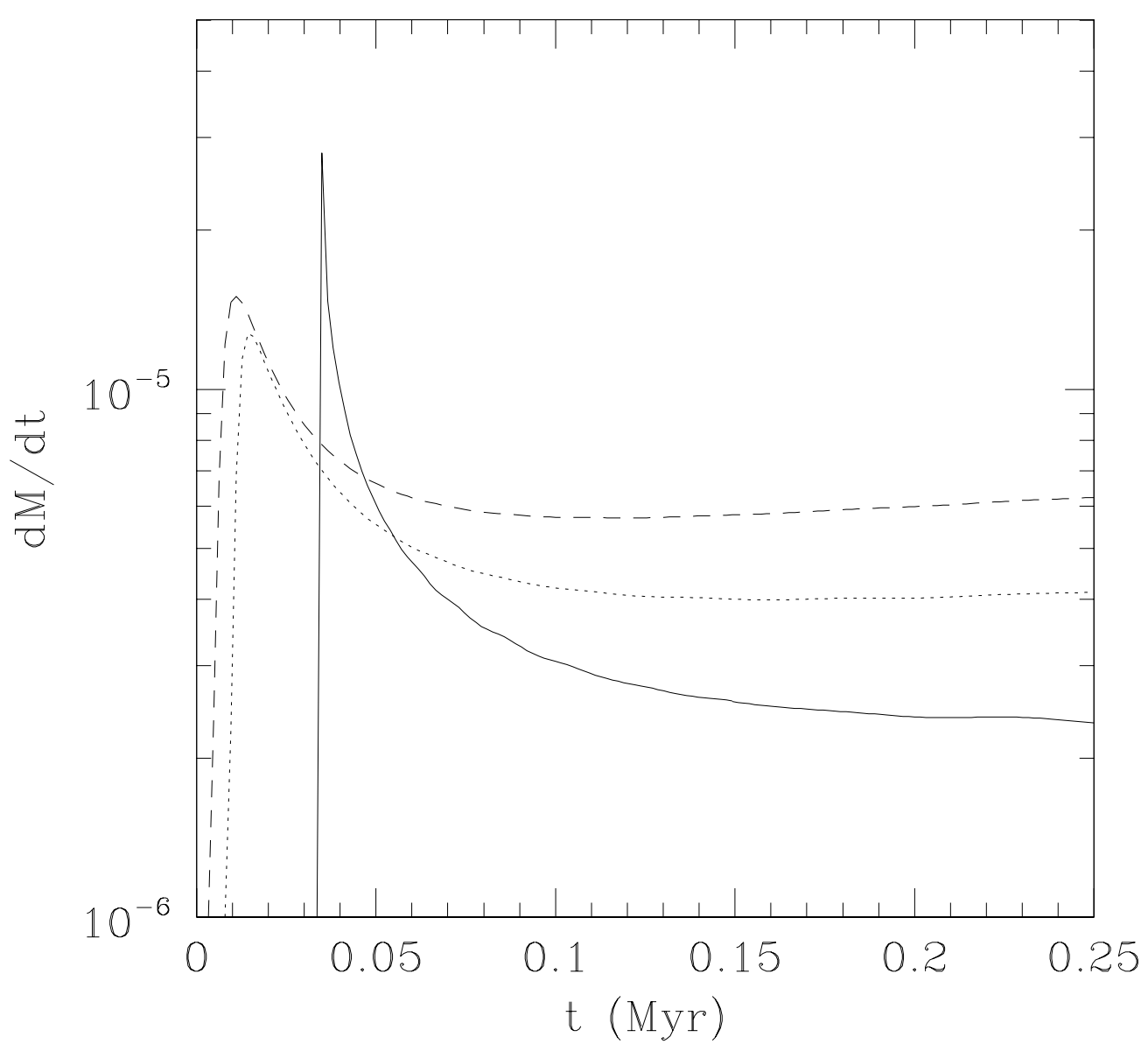

Fig. 13.- Mass infall rates for flat-topped cores as a function of time for varying initial infall speeds. This set of numerical collapse calculations begins with typical parameters observed in flat-topped cores with sound speed $a_{S}=0.2 \mathrm{~km} / \mathrm{s}$ and a central region of nearly constant density spanning $r_{C} \approx 10^{16} \mathrm{~cm}$. The initial states are overdense at the 10 percent level, so that $\mathcal{A}=2.2$. The solid curve shows the resulting mass infall rate (expressed in $M_{\odot} \mathrm{yr}^{-1}$ ) for the case with no initial velocity. The dotted (dashed) curve shows the corresponding mass infall rate for starting inward speeds of $u_{i n}=0.5 a_{S}\left(u_{i n}=a_{S}\right)$. When $u_{\text {in }} \neq 0$, the mass infall rates reach their peak values more quickly, the peak values are smaller, and the infall rates more rapidly approach their asymptotic values (as predicted by the similarity solutions). 